\title{
Flavor and stability of milk proteins
}

\author{
T. J. Smith, R. E. Campbell, Y. Jo, and M. A. Drake ${ }^{1}$ \\ Department of Food, Bioprocessing and Nutrition Sciences, Southeast Dairy Foods Research Center, North Carolina State University, \\ Raleigh 27695
}

\section{ABSTRACT}

A greater understanding of the nature and source of dried milk protein ingredient flavor(s) is required to characterize flavor stability and identify the sources of flavors. The objective of this study was to characterize the flavor and flavor chemistry of milk protein concentrates (MPC 70, 80, 85), isolates (MPI), acid and rennet caseins, and micellar casein concentrate (MCC) and to determine the effect of storage on flavor and functionality of milk protein concentrates using instrumental and sensory techniques. Spray-dried milk protein ingredients (MPC, MPI, caseins, MCC) were collected in duplicate from 5 commercial suppliers or manufactured at North Carolina State University. Powders were rehydrated and evaluated in duplicate by descriptive sensory analysis. Volatile compounds were extracted by solid phase microextraction followed by gas chromatography-mass spectrometry (GC-MS) and gas chromatography-olfactometry. Compounds were identified by comparison of retention indices, odor properties, and mass spectra against reference standards. A subset of samples was selected for further analysis using direct solvent extraction with solvent-assisted flavor extraction, and aroma extract dilution analysis. External standard curves were created to quantify select volatile compounds. Pilot plant manufactured MPC were stored at 3,25 , and $40^{\circ} \mathrm{C}$ ( $44 \%$ relative humidity). Solubility, furosine, sensory properties, and volatile compound analyses were performed at $0,1,3$, 6 , and 12 mo. Milk proteins and caseins were diverse in flavor and exhibited sweet aromatic and cooked/milky flavors as well as cardboard, brothy, tortilla, soapy, and fatty flavors. Key aroma active compounds in milk proteins and caseins were 2-aminoacetophenone, nonanal, 1-octen-3-one, dimethyl trisulfide, 2-acetyl-1-pyrroline, heptanal, methional, 1-hexen-3-one, hexanal, dimethyl disulfide, butanoic acid, and acetic acid. Stored milk

Received January 3, 2016.

Accepted February 25, 2016.

${ }^{1}$ Corresponding author: maryanne_drake@ncsu.edu proteins developed animal and burnt sugar flavors over time. Solubility of MPC decreased and furosine concentration increased with storage time and temperature. Solubility of MPC 80 was reduced more than that of MPC 45, but time and temperature adversely affected solubility of both proteins, with storage temperature having the greatest effect. Flavor and shelf stability of milk proteins provide a foundation of knowledge to improve the flavor and shelf-life of milk proteins.

Key words: milk protein, flavor, shelf life

\section{INTRODUCTION}

Milk protein concentrates (MPC) and isolates (MPI) are a relatively new category of dried dairy ingredients. Milk protein concentrate is a complete dairy protein ingredient containing both caseins and whey proteins at a concentration ranging from 40 to $89 \%$, whereas MPI contains a protein concentration of $90 \%$ or greater. Milk protein concentrates and isolates are manufactured by concentrating milk proteins (both whey proteins and caseins) from fluid skim milk by membrane filtration followed by spray drying. Globally, MPC production has quadrupled to over 164,000 t from 2000 to 2011 (USDEC, 2012). The United States is both the single largest user in the world and the single largest importer, and the US Dairy Export Council (USDEC) predicts significant growth through the end of the decade to satisfy rising demand. Micellar casein concentrate (MCC) is a product for which commercial production was initiated a few years ago so production data are not yet available. Micellar casein concentrate is produced by microfiltration of skim milk, followed by ultrafiltration to further concentrate proteins before spray drying. In the production of MCC, serum proteins are removed via microfiltration. In production of MPI, filtration is used to remove lactose from milk, but both casein and whey proteins are retained in their original proportions found in milk, without combining separately produced casein (caseinate) and whey proteins (ADPI, 2015). The flavor of these ingredients is an important quality parameter as ingredient flavor can influence consumer liking and acceptability of the finished product. 
Most of the research conducted on MPC, MCC, or caseins has been focused on functionality and very little research has been conducted on flavor or elucidating the compounds responsible for the characteristic flavors of these proteins. Functional characteristics such as solubility during storage (Le et al., 2011a), the effect of $\mathrm{NaCl}$ addition during diafiltration on solubility, hydrophobicity, and soluble protein composition (Mao et al., 2012; Sikand et al., 2013), change in protein structure upon oxidation (Dalsgaard et al., 2007), and the influence of the Maillard reaction on functionality (Le et al., 2011a; Thomas et al., 2004) have all been studied in recent years; however, the effects of low (45\%) and high $(80 \%)$ protein content, and low temperature storage $\left(3^{\circ} \mathrm{C}\right)$ on solubility and flavor have not been investigated.

Rennet and acid casein have been described as having an animal/wet dog or gluey flavor and characteristic aroma components of rennet casein were 2-aminoacetophenone, hexanoic acid, indole, guaiacol, and $p$-cresol (Karagul-Yuceer et al., 2003). The sensory profiles of higher protein MPC (MPC 77, MPC 80, and MPI) were characterized by tortilla, brothy, cardboard, and animal flavors (Drake et al., 2009). The flavor of MCC has not been reported, and the flavor, flavor chemistry, and flavor stability of milk proteins have not been fully investigated. The objectives of this study were to characterize the flavor and flavor chemistry of milk protein concentrates (MPC 70, 80, 85), isolates (MPI), acid and rennet caseins, and micellar casein concentrate (MCC; experiment 1), and to determine and compare the effects of storage temperature and time on the sensory and functionality (shelf-life) of low (45\%) and high (85\%) protein MPC over an extended period (1 yr; experiment 2) using instrumental and sensory techniques.

\section{MATERIALS AND METHODS}

\section{Experimental Overview}

Two experiments (experiments 1 and 2) were included in this study. The purpose of experiment 1 was to determine the sensory profiles and key aroma active volatile compounds in milk proteins. To carry out this objective, 9 commercial milk protein products were collected and one manufactured in the North Carolina State University (NCSU) dairy pilot plant. Products were collected or manufactured in duplicate. Descriptive analysis, headspace extraction and quantification of volatile compounds by GC-MS and gas chromatography-olfactometry (GC-O), and solvent-assisted flavor evaporation (SAFE) followed by aroma extract dilution analysis (AEDA) were performed on the dried milk protein products. The purpose of experiment 2
Table 1. Commercial proteins and manufacturers

\begin{tabular}{ll}
\hline Manufacturer & Protein type \\
\hline A & Rennet casein \\
A & Acid casein \\
B & MPC 70 \\
B & MPC 85 \\
B & MPI \\
C & MCC \\
D & MPC 80 \\
E & MPC 80 \\
E & MPC 85 \\
P & MPC 45 \\
P & MPC 80
\end{tabular}

${ }^{1}$ Two lots of each protein were obtained from each supplier. MPC $=$ milk protein concentrate; $\mathrm{MCC}=$ micellar casein concentrate; $\mathrm{MPI}=$ milk protein isolate. Letters A-E are commercial suppliers; P means manufactured in the North Carolina State University pilot plant.

was to determine the flavor and functional properties of milk protein concentrates across storage. To conduct this objective, MPC 45 and 80 were produced in the NCSU dairy pilot plant and stored at constant relative humidity $(44 \%)$ at 3,25 , or $40^{\circ} \mathrm{C}$. Samples were measured after $0,1,3,6$, and 12 mo. Descriptive analysis, furosine content, solubility, and flavor volatiles by GCMS were measured on all samples at each time point.

\section{Sample Collection}

Commercial Samples and Chemical Standards. Nine different products were obtained in duplicate lots from 5 different manufacturers across the United States and Canada (Table 1). Upon arrival, samples were store at $-80^{\circ} \mathrm{C}$ until analysis. Chemical standards were obtained from Sigma-Aldrich (Milwaukee, WI) and ChemStep (Montesquieu, France).

Pilot Plant Production of MPC. Raw skim milk was obtained from the North Carolina State University Dairy Research and Education Unit on 2 different occasions. The milk was HTST pasteurized $(720 \mathrm{~kg} / \mathrm{h}$; hold time $15 \mathrm{~s}$ at $72^{\circ} \mathrm{C}$ ) using a plate heat exchanger (model T4 RGS-16/2, SPX Flow Technology, Greensboro, NC). The milk was the ultrafiltered and diafiltered using a laboratory scale ultrafiltration unit (model Lab 46, Filtration Engineering, Champlin, MN) equipped with 4 spiral wound membranes (Snyder Filtration, Vacaville, CA; nominal cutoff: 10,000 Da; surface area $5.0 \mathrm{~m}^{2} /$ membrane). The temperature for ultrafiltration was $50^{\circ} \mathrm{C}$. For the MPC 45 , the skim milk was ultrafiltered and protein monitored by Sprint Rapid Protein Analyzer (CEM, Matthews, NC) and Smart System 5 moisture/solids analyzer (CEM) until $45 \%$ protein (wt/wt) was achieved. Total filtration time was approximately $25 \mathrm{~min}$. Total solids before spray drying were approximately 10\%. For MPC 80, diafiltration 
water was added at a rate of $40 \%$ of the original weight of the milk. Total filtration time was approximately $3 \mathrm{~h}$. Once the MPC reached 45 or $80 \%$ protein (wt/ wt), the MPC retentate was then spray dried (nozzle atomization, model Lab 1, Anhydro Inc., Soeberg, Denmark). Inlet temperature was $200^{\circ} \mathrm{C}$ and the outlet temperature was $90^{\circ} \mathrm{C}$. The MPC 80 was then weighed and separated into lots for flavor analysis or storage. The MPC 80 used in the flavor study were collected and stored in Mylar bags (Uline, Pleasant Prairie, WI) at $-80^{\circ} \mathrm{C}$ following production. The MPC 45 and 80 used in the storage study were weighed, and sealed in laminated poly-nylon vacuum pouches (30NV0608, Elkay Plastics, Austell, GA) at a relative humidity of $44 \%$, boxed (to protect from light exposure), and stored in temperature-controlled incubators at predetermined temperatures $\left(3,25\right.$, or $\left.40^{\circ} \mathrm{C}\right)$. Each pouch contained approximately $2 \mathrm{~kg}$ of powder such that a new pouch was opened and evaluated at each time point.

\section{Headspace Extraction of Volatile Compounds}

$\boldsymbol{G} \boldsymbol{C}$ - $\boldsymbol{M S}$. Volatile compounds in caseins, MPC/MPI, and MCC powders were extracted by solid-phase microextraction (SPME) and then separated and identified by GC-MS with selective ion monitoring using a modified method of Liaw et al. (2010). Volatile compounds for selective ion monitoring were selected from previous literature (Carunchia Whetstine et al., 2003; Karagul-Yuceer et al., 2003; Drake et al., 2009; Liaw et al., 2011; Campbell et al., 2013), GC-O (described below), and by evaluation of chromatograms collected in scan mode. Dried powders were reconstituted at 10\% solids (wt/vol). All samples contained $10 \%$ (wt/vol) sodium chloride (Fisher Scientific), and $10 \mu \mathrm{L}$ of internal standard solution (2-methyl-3-heptanone in methanol at $81 \mathrm{mg} / \mathrm{kg}$ (Sigma-Aldrich) in $20 \mathrm{~mL}$ autosampler vials with steel screw tops containing silicone septa faced in Teflon (Microliter Analytical, Suwanee, GA). Samples were injected using a CombiPal autosampler (CTC Analytics, Zwingen, Switzerland) attached to an Agilent 7820A GC with 5975B MSD (Agilent Technologies Inc., Santa Clara, CA). Samples were maintained at $5^{\circ} \mathrm{C}$ before fiber exposure. Samples were equilibrated at $40^{\circ} \mathrm{C}$ for $25 \mathrm{~min}$ before $30 \mathrm{~min}$ fiber exposure of a 1 $\mathrm{cm}$ of [divinylbenzene/carboxen/polydimethylsiloxane (DVB/CAR/PDMS); Supelco Inc.] fiber (Supelco, Bellefonte, PA) at $31 \mathrm{~mm}$ with $4 \mathrm{~s}$ of pulsed agitation at $250 \mathrm{rpm}$. Fibers were injected for $5 \mathrm{~min}$ at a depth of $50 \mathrm{~mm}$. Each sample from each lot was evaluated in triplicate.

The GC method used an initial temperature of $40^{\circ} \mathrm{C}$ for 3 min with a ramp rate of $10^{\circ} \mathrm{C} / \mathrm{min}$ to $250^{\circ} \mathrm{C}$ held for $5 \mathrm{~min}$. The SPME fibers were introduced into the split/splitless injector at $250^{\circ} \mathrm{C}$. A ZB- $5 \mathrm{~ms}$ column (ZB-5ms $30 \mathrm{~m}$ length $\times 0.25 \mathrm{~mm}$ i.d. $\times 0.25 \mu \mathrm{m}$ film thickness; Phenomenex, Torrance, CA) was used for all analyses at a constant flow rate of $1 \mathrm{~mL} / \mathrm{min}$. Purge time was set at $1 \mathrm{~min}$. The MS transfer line was maintained at $250^{\circ} \mathrm{C}$ with the quad at $150^{\circ} \mathrm{C}$ and source at $250^{\circ} \mathrm{C}$. Compounds were identified using the NIST 2005 library of spectra and comparison of spectra of authentic standards injected under identical conditions. Relative abundance for each compound was calculated using the calculated recovery of the internal standard concentration to determine relative concentrations of each compound. Retention indices were calculated using an alkane series (Sigma Aldrich; Vandendool and Kratz, 1963).

$\boldsymbol{G C}-\boldsymbol{O}$. Solid-phase microextraction followed by GC-O for characterization of aroma active compounds was conducted on all caseins, MPC/MPI (time 0 only), and MCC. Twenty milliliters of each sample $(10 \%$ solids wt/vol) along with $10 \%$ sodium chloride (wt/ wt) was dispersed into six 40-mL amber vials $(28 \times$ $98 \mathrm{~mm}$; Supelco Inc.) with a polytetrafluoroethylene/ silicone septum (Supelco Inc.) and a stir bar. The vials were heated at $40^{\circ} \mathrm{C}$ for 20 min with constant stirring. A SPME fiber (DVB/CAR/PDMS, Supelco Inc.) was exposed in each sample at $1 \mathrm{~cm}$ for $1 \mathrm{~h}$. The fiber was then injected on an Agilent 6850 gas chromatographyflame-ionization detector (FID) equipped with an olfactometer port (Agilent Technologies Inc.). The GC method used an initial temperature of $40^{\circ} \mathrm{C}$ for $3 \mathrm{~min}$. The temperature was then increased at a rate of $10^{\circ} \mathrm{C} /$ min to $150^{\circ} \mathrm{C}$, followed by $30^{\circ} \mathrm{C} / \mathrm{min}$ to $200^{\circ} \mathrm{C}$, and held for $5 \mathrm{~min}$. Samples were evaluated in duplicate on 2 different columns: polar ZB-WAX $(30-\mathrm{m}$ length $\times 0.25$ $\mathrm{mm}$ i.d. $\times 0.25-\mu \mathrm{m}$ film thickness; Zebron; Phenomenex Inc.) and a nonpolar ZB-5ms (30-m length $\times 0.25-\mathrm{mm}$ i.d. $\times 0.25-\mu \mathrm{m}$ film thickness; Zebron; Phenomenex Inc.). Effluent was split 1:1 between the FID and sniffing port using deactivated fused-silica capillaries (1-m length $\times$ 0.25-mm i.d.; Phenomenex Inc.). The FID sniffing port was held at a temperature of $300^{\circ} \mathrm{C}$, with a helium carrier gas flow of $1,018.6 \mathrm{~cm} / \mathrm{s}$, and the port was supplied with humidified air at $30 \mathrm{~mL} / \mathrm{min}$. Each sample was evaluated on each column by at least 2 highly experienced sniffers (each with $>50$ h previous experience with GC-O) who recorded aroma character and perceived intensity.

\section{Solvent Extraction of Volatile Compounds}

Direct Solvent Extraction. A representative subset of milk proteins $(\mathrm{n}=8$; manufacturer A: rennet casein, acid casein, manufacturer B: MPC 85, MPI, manufacturer C: MCC, manufacturer D: MPC 80, manufacturer 
E: MPC 80, MPC 85, duplicate lots) was selected based on sensory and SPME results for further analysis via direct solvent extraction with SAFE. Direct solvent extraction was conducted on powders following the methods of Evans et al. (2009). Direct solvent extraction was performed in duplicate on each selected sample. One hundred grams of powder was divided among two 250-mL Teflon bottles with Tefzel closures (Nalgene, Rochester, NY). Thirty milliliters of water was added to each bottle and was allowed to rehydrate for $30 \mathrm{~min}$. Then, $100 \mathrm{~mL}$ of diethyl ether (EMD Chemicals Inc., Gibbstown, NJ) was added to the powder/water mixture along with $20 \mu \mathrm{L}$ of internal standard at $81 \mathrm{mg} / \mathrm{kg}$ each (2-methyl-3-heptanone, 2-methyl-pentanoic acid in ether, Sigma Aldrich). The bottles were agitated using a Roto mix (Tyle 50800, Thermolyne, Dubuque, IA) on high speed for $30 \mathrm{~min}$. The solvent phase was separated from the mixture by low-speed centrifugation $(1,450 \times g)$ for $10 \mathrm{~min}$ at $10^{\circ} \mathrm{C}$. After centrifugation, the ether phase containing the volatile components was collected into an amber glass jar with a Teflon-lined lid. Diethyl ether addition, agitation, and centrifugation were repeated 3 more times each with $50 \mathrm{~mL}$ of diethyl ether added to each bottle each time. The extracts were then concentrated under a stream of nitrogen gas to $200 \mathrm{~mL}$. Solvent extracts were stored at $-20^{\circ} \mathrm{C}$.

$\boldsymbol{S A F E}$. Solvent extracts were distilled by SAFE (Ace Glassware, Vineland, NJ). The assembly was similar to the methods of Evans et al. (2009) and Whitson et al. (2011). The glass SAFE head, with the 2-L roundbottom flask attached, was connected to 2 glass traps. The primary trap for receiving the final distilled solvent extract and the secondary trap for waste were both submerged in liquid nitrogen via separate Dewar flasks. The round bottom flask was kept at $50^{\circ} \mathrm{C}$ using a water bath. Vacuum was reached in the glassware using a rough pump/diffusion pump combination. The solvent extract was poured into the top of the SAFE head and introduced into the vacuum system drop-wise until all of the sample was introduced. Distillation was carried out for 10 min under vacuum $(1.33 \mathrm{kPa})$ and kept at $50^{\circ} \mathrm{C}$ with a circulating water bath. After distillation, the sample was concentrated under a stream of nitrogen gas to $20 \mathrm{~mL}$ and transferred to a $50-\mathrm{mL}$ screw-top glass tube for phase separation.

Phase Separation. To separate solution into neutral/basic and acid fractions, the concentrated distillate was first washed twice with $3 \mathrm{~mL}$ of sodium bicarbonate (0.5 M; Fisher Scientific) and mixed vigorously. The concentrate was washed a total of 3 times, and after each wash, the bottom layer (water phase) was placed into another glass tube. The resulting concentrate was the neutral/basic fraction and was dried over anhydrous sodium sulfate (VWR International) to remove any residual water. The neutral/basic fraction was then concentrated to $0.5 \mathrm{~mL}$ under a stream of nitrogen gas. The water phase was acidified by adding hydrochloric acid (18\% wt/vol; Sigma Aldrich) until a pH of 2 to 2.5 was reached. Acidic volatiles were then extracted by the addition of $5 \mathrm{~mL}$ of diethyl ether, mixing vigorously, and removing the top ether phase. This process was repeated 2 more times. The acidic volatiles were then dried over anhydrous sodium sulfate and concentrated to $0.5 \mathrm{~mL}$ under a stream of nitrogen gas.

\section{Volatile Analysis of Solvent Extracts}

$\boldsymbol{G C}$-MS. Selected volatile compounds in solvent extraction fractions were detected using both scan and selective ion monitoring simultaneously. Analysis was conducted on an Agilent 7890B with a 5977A mass selector detection (Agilent Technologies Inc.). Separations were performed on a fused silica capillary column Zb-5ms $30 \mathrm{~m}$ length $\times 0.25 \mathrm{~mm}$ i.d. $\times 0.25 \mu \mathrm{m}$ film thickness; Phenomenex Inc.). Helium was used as the carrier gas at a constant flow of $1.5 \mathrm{~mL} / \mathrm{min}$. The GC-MS oven program started with an initial oven temperature of $40^{\circ} \mathrm{C}$ for $3 \mathrm{~min}$, increasing to $90^{\circ} \mathrm{C}$ at a rate of $10^{\circ} \mathrm{C}$ per min, then increasing $5^{\circ} \mathrm{C}$ per min to $200^{\circ} \mathrm{C}$, then $20^{\circ} \mathrm{C}$ per min to $250^{\circ} \mathrm{C}$ hold for $5 \mathrm{~min}$ and a final hold time of $10 \mathrm{~min}$. The MSD conditions included capillary direct interface temperature, $280^{\circ} \mathrm{C}$; source temperature $230^{\circ} \mathrm{C}$, quad temperature $150^{\circ} \mathrm{C}$. The injector temperature was $250^{\circ} \mathrm{C}$.

One microliter of each solvent extract was injected in duplicate in the pulsed splitless $(172.4 \mathrm{kPa}$ air for 0.5 min) mode. A 3.75-min solvent delay was programmed into the MS acquisition parameters for solvent injections. Retention indices were calculated using an alkane series (Sigma Aldrich; Vandendool and Kratz, 1963).

$\boldsymbol{G C}-\boldsymbol{O}$ with $\boldsymbol{A E D A}$. One microliter of solvent extract was injected into the GC injection port, maintained at $250^{\circ} \mathrm{C}$, fitted with a direct injection liner $(\mathrm{Su}-$ pelco; Sigma Aldrich). Column conditions and GC-O parameters were identical to those described previously for SPME. Both neutral/basic and acidic fractions from solvent extracts were analyzed in duplicate on both columns. Postpeak intensity of aroma active compounds were evaluated and recorded by 2 experienced sniffers, each with $>100 \mathrm{~h}$ experience with GC-O of dairy proteins.

The AEDA was conducted on solvent extracts under similar conditions as those used for postpeak intensity GC-O. A step-wise dilution of 1:3 ( $\mathrm{vol} / \mathrm{vol})$ with diethyl ether was followed until 2 experienced sniffers detected no odorants. The neutral/basic fractions were injected onto the nonpolar $\mathrm{Zb}-5 \mathrm{~ms}$ capillary column and acid fractions were injected onto the polar ZB-WAX capil- 
lary column. The highest dilution, for which an aroma was still detected, was reported as the flavor dilution factor.

\section{Compound Identification and Quantitation}

Mass spectra, retention index, and aroma properties of authentic standards injected under identical conditions on the GC-O and GC-MS were used for aroma active compound identification. Nineteen compounds, 9 of which were fatty acids, were selected for quantification using external standard curves. These compounds were selected due to their high $\log _{3}$ flavor dilution (FD) values, aroma character, and previous literature (Karagul-Yuceer et al., 2003; Drake et al., 2009). Due to retention times and the mandatory solvent delay on the GCMS after SAFE, 3 of these compounds [hexanal, 1-hexen-3-one, and dimethyl disulfide (DMDS)] were quantified via SPME. The remaining 16 compounds [2-acetylpyroline, 1-octen-3-one, 2-aminoacetophenone, heptanal, nonanal, dimethyl trisulfide (DMTS), methional, and butyric, pentanoic, hexanoic, heptanoic, octanoic, nonanoic, decanoic, dodecanoic, and tetradecanoic acids] were quantified by SAFE. Quantification of selected volatile compounds in SAFE extracts and SPME vials was executed using 5-point external standard curves ranging from $0.50 \mu \mathrm{g} / \mathrm{kg}$ to $2 \mathrm{mg} / \mathrm{kg}$ (depending on selected compound) and integrated to an internal standard $[81 \mathrm{mg} / \mathrm{kg} 2$-methyl-3-heptanone, 2-methyl-pentanoic acid in ether (SAFE extracts) or water (SPME)]. For SAFE extracts, external standard curves were prepared by adding selected compounds to $30 \mathrm{~mL}$ of water, which was then added to $50 \mathrm{~g}$ of powder in a $250-\mathrm{mL}$ Teflon bottles with Tefzel closures (Nalgene) and held at $21^{\circ} \mathrm{C}$ for $30 \mathrm{~min}$. Direct solvent extraction, SAFE, and phase separation proceeded as previously described. Extracts were injected onto the GC-MS using previously described parameters. For SPME, the selected compounds were diluted in water and then added to the milk protein powder, prepared as described previously. Relative abundance of other selected compounds was calculated relative to internal standard recovery.

\section{Descriptive Analysis}

Descriptive sensory analysis was conducted on MPC/ MPI, MCC, and caseins using a trained descriptive sensory panel and an established dairy flavor language (Drake et al., 2003, 2009). Panelists $(\mathrm{n}=8)$ each had more than $50 \mathrm{~h}$ of training and $150 \mathrm{~h}$ of previous experience with the sensory analysis of dried dairy ingredients using the Spectrum descriptive analysis method (Meilgaard et al., 2009). All sensory testing was conducted in accordance with NCSU Institutional Review Board for Human Subjects guidelines.

Powders were rehydrated to $10 \%$ solids (wt/vol) with deionized water and dispensed into 3-digit-coded 60-mL lidded cups for evaluation (Solo Cup Company, Champaign, IL). Preparations were conducted with overhead lights off to avoid exposure to light. Samples were evaluated by each panelist in duplicate. Sensory data were collected using Compusense Five, release 4.8 (Compusense, Guelph, Canada).

\section{Solubility Analysis}

Solubility analysis was performed on MPC 45 and MPC 85 produced in the NCSU pilot plant at all storage time points. Solutions of $10 \%$ protein (wt/vol) were made by rehydrating the MPC to $80 \%$ of the total volume desired, for $6 \mathrm{~h}$ at room temperature on a stir plate set to $300 \mathrm{rpm}$ (Campbell et al., 2011) and refrigerated overnight at $4 \mathrm{C}$. The solutions were then equilibrated to $25^{\circ} \mathrm{C}$ and the $\mathrm{pH}$ was adjusted using $1 \mathrm{~N} \mathrm{NaOH}$ to a $\mathrm{pH}$ of 7 and brought to a total volume of $100 \mathrm{~mL}$ with deionized water, resulting in $10 \%$ (wt/vol) solids solutions. Total protein was measured on the uncentrifuged solutions. Solutions were then centrifuged at $16,500 \times$ $g$ for 10 min at $10^{\circ} \mathrm{C}$ using a model RC5B centrifuge (Thermo Scientific) and the supernatants were again measured for protein concentration (solubility). Protein concentration was measured by the micro-BCA assay using a kit from Thermo Fisher Scientific/Pierce (Rockford, IL). Protein solutions before and after centrifugation were diluted 1:100 in deionized water. Solutions were added to working reagent in a ratio of $1: 8$ and pipetted in triplicate into a 96 -well plastic plate. Deionized water with working solution was used as a reference blank. The plate was put on a shaker for $30 \mathrm{~s}$ and incubated at $37 \mathrm{C}$ for $30 \mathrm{~min}$. The plate was brought to room temperature and read on a Tecan Safire plate reader spectrophotometer at wavelength $562 \mathrm{~nm}$ (Tecan, Durham, NC). Analysis was performed in quadruplicate. Solubility was calculated using the following equation (where Abs = absorbance):

$$
\begin{gathered}
\text { protein solubility }=100-\left\{\left[\left(\mathrm{Abs}_{\text {before }}-\mathrm{Abs}_{\text {after }}\right) /\right.\right. \\
\left.\left.\mathrm{Abs}_{\text {before }}\right] \times 100\right\} .
\end{gathered}
$$

\section{Furosine Analysis}

Furosine analysis, adapted from Baptista and Carvalho (2004), was performed on MPC 45 and MPC 85 at each time point. Approximately $30 \mathrm{mg}$ of MPC was placed into a screw-cap (polytetrafluoroethylene-faced septa) glass tube (VWR). Water (HPLC grade, $2.5 \mathrm{~mL}$, 
EMD Chemicals) was added and vortexed. Two milliliters of $8 \mathrm{~N} \mathrm{HCl}$ (EMD Chemicals) was added and mixed well. The solution was placed into an oven at $110^{\circ} \mathrm{C}$ and incubated for $24 \mathrm{~h}$. A $400-\mu \mathrm{L}$ aliquot was placed into a glass tube and the $\mathrm{HCl}$ was blown down with nitrogen until completely dry. Two milliliters of HPLC water was added and mixed. The solution was then filtered through a $0.45-\mu \mathrm{m}$ syringe filter into an HPLC vial (Phenomenex). Furosine was quantified by HPLC (Breeze HPLC, Waters, Milford, MA) using a mobile phase of $0.06 M$ sodium acetate, $\mathrm{pH} 4.3$ with acetic acid at a flow rate $1 \mathrm{~mL} / \mathrm{min}$ pumped through a binary pump (Waters 1525, Waters). Twenty microliters of sample was injected (Waters 2707 autosampler) onto the column (Lunca $5 \mathrm{u} \mathrm{C8} 100 \mathrm{~A} 250 \times 4.6 \mathrm{~mm}$ ) at $35^{\circ} \mathrm{C}$. Sample was analyzed using a photodiode array detector (Waters 2998) with a maxima of $280 \mathrm{~nm}$. Sample run time was $10 \mathrm{~min}$. All MPC were extracted and evaluated in triplicate. Furosine increase was determined by calculating the percent increase of furosine concentration in the stored MPC to the furosine concentration of the time 0 MPC.

\section{Statistical Analysis}

Data in experiment 1 were analyzed by a one-way ANOVA using a general linear model with Fisher's least significant difference for means separation (XLSTAT, version 2015.1.01; Addinsoft Inc., New York, NY). All data in experiment 2 were analyzed by 3 -way ANOVA (protein $\times$ temperature $\times$ storage) with Fisher's least significant difference for means separation (XLSTAT, version 2015.1.01; Addinsoft Inc.).

\section{RESULTS AND DISCUSSION}

\section{Experiment 1}

Instrumental Volatile and Descriptive Analysis. Forty-two odor active events were detected in dried milk proteins by SPME GC-O. Of the 42, 30 were identified by aroma, retention index, and mass spectra, and 12 were tentatively identified by retention index on ZB5 or wax column and odor quality (Table 2). Predominant odorants of the MPC, MCC, and casein samples were also identified by solvent extraction with AEDA. Forty-three odor active events were detected in dried milk proteins by solvent extraction with AEDA. Twenty-two neutral/basic and acid odorants were identified by retention index, aroma, and mass spectra, with 16 tentatively identified by retention index and odor quality, and 5 unknowns (Table 3). All of the odor active compounds listed in Tables 2 and 3 were previously reported as odor-active components of milk, cheese, casein, skim milk powder (SMP), fluid whey, whey powder, whey protein concentrates, or whey protein hydrolysates (Hammond and Hill, 1964; Preininger and Grosch, 1994; Shiratsuchi et al., 1994a,b; KaragulYuceer and Cadwallader, 2001; Karagul-Yuceer et al., 2002, 2003; Mahajan et al., 2004; Carunchia Whetstine et al., 2005; Kobayashi et al., 2008; Leksrisompong et al., 2010).

Solvent extraction and AEDA of milk proteins and caseins detected many compounds not detected by SPME headspace extraction. These volatile compounds included 2-methyl-1-propanol, heptanal, 1-octen-3-one, E,E 2,4 heptadienal, 2-acetyl thiazole, benzyl alcohol, gamma terpinene, 2-ethyl-3,5-dimethylpyrazine, sotolon, homofuraneol, 2,4-dimethylphenol, 4-ethylphenol, decanal, E,Z-2,4-nonadienal, benzothiazole, acetic acid, $\beta$ ionone, propionic acid, 5-methyl furfural, butanoic, pentanoic, hexanoic, and decanoic acids, and 1-octadecanol. Similarly, SPME headspace extraction detected many compounds not detected by solvent extraction (SAFE) and AEDA of milk proteins and caseins. No volatile extraction approach is unbiased. Headspace extraction generally recovers more highly volatile compounds, whereas solvent extraction tends to favor higher molecular weight compounds. For these reasons, both extraction approaches were applied in the current study.

The greatest number of odorants (neutral/basic and acid) were identified in acid and rennet caseins. Acidic odorants consisted mainly of short-chain volatile acids (Table 3). On the basis of $\log _{3}$ FD (>2) factors, the most potent odorants in rennet casein were 1-hexen3-one, heptanal, methional, 2-acetyl-1-pyrroline, DMTS, 1-octen-3-one, octanal, 2-methoxyphenol, benzothiazole, acetic, propionic, butanoic, and pentanoic acids, and 1-octadecanol. In acid casein, compounds with high $\log _{3}$ FD factors included hexanal, homofuraneol, methional, 1-octen-3-one, and butanoic acid. The compounds with the highest $\log _{3}$ FD factors in milk proteins (MPC 70,80, 85, MCC, and MPI) were dimethyl disulfide, hexanal, 1-hexen-3-one, sotolon, homofuraneol, 4-hexen-3-one, heptanal, methional, 1-cyclohexen-3-one, 2-acetyl-1-pyrroline, dimethyl trisulfide, 1-octen-3-one, octanal, guaiacol, nonanal, decanal, benzothiazole, 2-aminoacetophenone, gammadecalactone, acetic, propionic, butanoic, pentanoic, and hexanoic acids, and delta-dodecalactone.

Among the MPC, MPI, and MCC, the MPC had the greatest number of neutral/basic and acid odorants identified with high $(>2) \log _{3}$ FD factors. Volatile compounds with the highest $\log _{3}$ FD factors $(>3.5)$ in MPC were dimethyl disulfide, hexanal, 2-acetyl-pyrroline, 1-octen-3-one, acetic acid, 2-methoxy phenol (guaiacol), nonanal, methional, 2-methyl-1-propanal, and 
Table 2. Odor-active compounds detected in caseins, milk protein concentrates (MPC), milk protein isolates (MPI), and micellar casein concentrates (MCC) by gas chromatographyolfactometry using solid-phase microextraction (SPME)

\begin{tabular}{|c|c|c|c|c|c|c|c|c|c|c|c|c|c|c|}
\hline $\begin{array}{l}\text { RI } \\
(\mathrm{ZB} 5)^{1}\end{array}$ & $\begin{array}{c}\mathrm{RI} \\
(\mathrm{WAX})^{2}\end{array}$ & $\begin{array}{l}\text { Method } \\
\text { of } \mathrm{ID}^{3}\end{array}$ & Description & Compound & $\begin{array}{c}\text { A: } \\
\text { Acid } \\
\text { casein }\end{array}$ & $\begin{array}{c}\text { A: } \\
\text { Rennet } \\
\text { casein }\end{array}$ & $\begin{array}{c}\text { B: } \\
\text { MPC } \\
70\end{array}$ & $\begin{array}{l}\text { B: } \\
\text { MPI }\end{array}$ & $\begin{array}{l}\text { B: } \\
\text { MPC } \\
85\end{array}$ & $\begin{array}{l}\mathrm{C}: \\
\mathrm{MCC}\end{array}$ & $\begin{array}{c}\text { D: } \\
\text { MPC } \\
80\end{array}$ & $\begin{array}{c}\text { E: } \\
\text { MPC } \\
85\end{array}$ & $\begin{array}{c}\text { E: } \\
\text { MPC } \\
80\end{array}$ & $\mathrm{PP}$ \\
\hline 588 & 667 & $\mathrm{RI}, \mathrm{O}, \mathrm{MS}$ & Pumpkin & Dimethyl sulfide & + & + & + & + & + & + & + & & & + \\
\hline 627 & 975 & $\mathrm{RI}, \mathrm{O}, \mathrm{MS}$ & Butter & Diacetyl & + & + & + & + & + & + & + & + & + & + \\
\hline 654 & & $\mathrm{RI}, \mathrm{O}$ & Metallic/cabbage & Methyl ethyl sulfide & & & & & & & + & & & + \\
\hline 662 & 912 & $\mathrm{RI}, \mathrm{O}, \mathrm{MS}$ & Cocoa/malty & 2-Methyl butanal & + & & + & & & + & & & & + \\
\hline 672 & & $\mathrm{RI}, \mathrm{O}, \mathrm{MS}$ & Sulfur/cabbage & Dimethyl disulfide & + & + & & & & & + & & & \\
\hline 678 & & $\mathrm{RI}, \mathrm{O}, \mathrm{MS}$ & Malty/burnt & 3-Methyl butanal & + & & + & + & & + & + & + & & \\
\hline 780 & & $\mathrm{RI}, \mathrm{O}$ & Fruity & Ethyl 2-methylpropanoate & & + & & & & & + & & & \\
\hline 809 & 1087 & RI, O, MS & Burnt & 1-Hexen-3-one & & & & & & & & + & & \\
\hline 832 & 1079 & $\mathrm{RI}, \mathrm{O}, \mathrm{MS}$ & Green & Hexanal & + & + & + & + & + & + & + & + & & + \\
\hline 845 & & $\mathrm{RI}, \mathrm{O}, \mathrm{MS}$ & Mint & 1-Hexanol & & & & & & 1 & 1 & + & & 1 \\
\hline 883 & 1189 & RI, O & Fruity & Ethyl-3-methylbutanoate & + & & & & & & + & & & \\
\hline 890 & 1316 & RI, O, MS & Nutty & Furfuryl alcohol & & + & & + & & & & & & + \\
\hline 902 & & $\mathrm{RI}, \mathrm{O}, \mathrm{MS}$ & Cooked & 2-Heptanone & + & + & + & & + & + & + & & + & + \\
\hline 931 & 1178 & $\mathrm{RI}, \mathrm{O}, \mathrm{MS}$ & Fishy & Z-4-Heptenal & + & & & + & & & & & + & \\
\hline 935 & 1465 & $\mathrm{RI}, \mathrm{O}, \mathrm{MS}$ & Potato & Methional & + & + & + & + & & + & + & + & & \\
\hline 948 & & $\mathrm{RI}, \mathrm{O}$ & Brothy & Dimethyl sulfone & & & & & & & + & + & & \\
\hline 955 & 1345 & $\mathrm{RI}, \mathrm{O}, \mathrm{MS}$ & Popcorn & 2-Acetyl pyrroline & + & + & + & + & + & & + & + & + & + \\
\hline 997 & 1379 & $\mathrm{RI}, \mathrm{O}, \mathrm{MS}$ & Sulfur/cabbage & Dimethyl trisulfide & + & + & & + & + & + & & + & & + \\
\hline 1009 & 1305 & $\mathrm{RI}, \mathrm{O}, \mathrm{MS}$ & Mushroom/metallic & 1-Octen-3-ol & + & + & + & + & + & + & + & + & + & + \\
\hline 1040 & 1274 & $\mathrm{RI}, \mathrm{O}, \mathrm{MS}$ & Citrus & Octanal & + & + & + & + & + & & + & + & + & + \\
\hline 1102 & & $\mathrm{RI}, \mathrm{O}, \mathrm{MS}$ & Caramel & Maltol & & & & & & & + & + & + & \\
\hline 1109 & & $\mathrm{RI}, \mathrm{O}, \mathrm{MS}$ & Green/vegetative & 3-Nonenal & & & + & & & + & + & 1 & 1 & \\
\hline 1119 & & $\mathrm{RI}, \mathrm{O}, \mathrm{MS}$ & Burnt/smoky & Guaiacol & + & + & + & + & + & + & + & + & + & + \\
\hline 1126 & & $\mathrm{RI}, \mathrm{O}, \mathrm{MS}$ & Green/fatty & Nonanal & + & & & & & & & + & + & \\
\hline 1139 & & $\mathrm{RI}, \mathrm{O}$ & $\begin{array}{l}\text { Cardboard/fatty/ } \\
\text { carpet }\end{array}$ & 2,6-Dimethylphenol & + & + & + & + & + & & + & + & & + \\
\hline 1171 & & $\mathrm{RI}, \mathrm{O}, \mathrm{MS}$ & Cardboard & Z-2-Nonenal & + & & & & & & & + & & \\
\hline 1190 & & $\mathrm{RI}, \mathrm{O}, \mathrm{MS}$ & Green & E,Z-2,6-Nonadienal & + & & + & & & & + & + & + & + \\
\hline 1196 & 1536 & $\mathrm{RI}, \mathrm{O}$ & Cucumber & E-2-Nonenal & + & & & + & & + & + & + & & \\
\hline 1204 & & $\mathrm{RI}, \mathrm{O}, \mathrm{MS}$ & Carpet & 2,3-Dimethylphenol & & + & & & + & & + & & + & \\
\hline 1240 & & $\mathrm{RI}, \mathrm{O}$ & Cooked burnt & 2,4,6-Trimethylphenol & + & & + & + & & + & + & + & & + \\
\hline 1248 & & RI, O & Fatty brothy & E,E-2,4-Nonadienal & & & & & + & & & + & & \\
\hline 1458 & & RI, O, MS & Green & E-2-Decenal & + & & & & & + & + & + & & \\
\hline 1344 & & RI, O & Licorice & Eugenol & & + & + & & + & & & & + & \\
\hline 1354 & $>2000$ & RI, O, MS & Corn chips & 2-Aminoacetophenone & + & + & + & & + & & + & + & + & + \\
\hline 1358 & & RI, O, MS & Soapy & E-2-Undecenal & + & & & & & & & & & \\
\hline 1366 & & RI, O & Fatty/corn chips & E,Z-2,4 Decadienal & + & + & & + & + & & + & + & + & \\
\hline 1379 & & $\mathrm{RI}, \mathrm{O}, \mathrm{MS}$ & Fatty/corn chips & E,E-2,4-Decadienal & + & + & & + & + & & + & + & + & \\
\hline 1476 & & $\mathrm{RI}, \mathrm{O}, \mathrm{MS}$ & Green & E-2-dodecenal & & & & & & + & & + & & \\
\hline 1578 & $>2000$ & $\mathrm{RI}, \mathrm{O}, \mathrm{MS}$ & Sweet & Delta-decalactone & & + & & & & & & & + & + \\
\hline 1602 & & RI, O & Minty & Alpha-curcumene & & & & & & & + & & & \\
\hline 1663 & & RI, O & Sweet & Delta-undecalactone & & & & + & & & & & & \\
\hline 1711 & & $\mathrm{RI}, \mathrm{O}, \mathrm{MS}$ & Fruity/sweet & Delta-dodecalactone & & & & & & & + & & & \\
\hline
\end{tabular}

${ }^{1}$ Retention indices (RI) from gas chromatography-olfactometry (GC-O) data on the ZB-5 column.

2. RI from GC-O data on the WAX column.

${ }^{3}$ Method of identification by RI, O (olfactometry), and MS (mass spectrometry) compared with authentic standards. 
¿ Table $3 . \log _{3} \mathrm{FD}$ (flavor dilution) values of odor-active compounds detected in caseins, milk protein concentrates (MPC), milk protein isolates (MPI), and micellar casein

\begin{tabular}{|c|c|c|c|c|c|c|c|c|c|c|c|c|c|c|}
\hline & $\begin{array}{c}\mathrm{RI}^{1} \\
\text { WAX }\end{array}$ & $\begin{array}{l}\mathrm{RI}^{1} \\
\text { DB5 }\end{array}$ & Fraction $^{2}$ & Compound & Odor $^{3}$ & $\mathrm{ID}^{4}$ & $\begin{array}{c}\text { A: } \\
\text { Acid } \\
\text { casein }\end{array}$ & $\begin{array}{c}\text { A: } \\
\text { Rennet } \\
\text { casein }\end{array}$ & $\begin{array}{c}\text { B: } \\
\text { MPC } \\
70\end{array}$ & $\begin{array}{l}\text { B: } \\
\text { MPC } \\
85\end{array}$ & $\begin{array}{l}\text { B: } \\
\text { MPI }\end{array}$ & $\begin{array}{c}\text { C: } \\
\text { MCC }\end{array}$ & $\begin{array}{c}\text { D: } \\
\text { MPC } \\
80\end{array}$ & $\begin{array}{l}\text { E: } \\
\text { MPC } \\
80\end{array}$ \\
\hline 1 & $\mathrm{ND}^{5}$ & 718 & $\mathrm{NB}$ & Dimethyl disulfide & Sulfur & $\mathrm{O}, \mathrm{RI}$ & 2 & $<1$ & $<1$ & $<1$ & $\mathrm{ND}$ & $<1$ & 6 & $<1$ \\
\hline 2 & ND & 802 & NB & Hexanal & Green & $\mathrm{O}, \mathrm{RI}, \mathrm{MS}$ & 3 & 2 & $<1$ & $<1$ & 2 & $<1$ & 4 & $<1$ \\
\hline 3 & ND & 809 & NB & 1-Hexene-3-one & Skunky/smoky & $\mathrm{O}, \mathrm{RI}$ & 2 & 4 & $<1$ & $<1$ & $<1$ & ND & 3 & $<1$ \\
\hline 4 & 1087 & ND & A & 2-Methyl-1-propanol & Burning plastic & $\mathrm{O}, \mathrm{RI}$ & 3 & 2 & $<1$ & 4 & 3 & ND & 3 & $<1$ \\
\hline 5 & ND & 811 & NB & Unknown & Mucus & $\mathrm{O}, \mathrm{RI}$ & $<1$ & $<1$ & 2 & 3 & $<1$ & 2 & ND & $<1$ \\
\hline 6 & 1025 & 865 & $\mathrm{~A} / \mathrm{NB}$ & Ethyl-3-methylbutanoate & Sweet/citrus & $\mathrm{O}, \mathrm{RI}$ & 2 & 2 & $<1$ & $<1$ & 2 & $<1$ & $<1$ & 2 \\
\hline 7 & ND & 894 & NB & Heptanal & Rubber/plastic & $\mathrm{O}, \mathrm{RI}, \mathrm{MS}$ & $<1$ & 3 & $<1$ & 3 & $<1$ & $<1$ & $<1$ & 2 \\
\hline 8 & 1241 & 906 & $\mathrm{~A} / \mathrm{NB}$ & Z-4-heptenal & Fishy & $\mathrm{O}, \mathrm{RI}$ & $<1$ & 2 & $<1$ & $<1$ & 2 & $<1$ & $<1$ & $<1$ \\
\hline 9 & ND & 911 & NB & Methional & Potato & $\mathrm{O}, \mathrm{RI}$ & 3 & 3 & 2 & 4 & 3 & ND & 3 & 3 \\
\hline 10 & ND & 921 & $\mathrm{NB}$ & Unknown & Carpet & $\mathrm{O}, \mathrm{RI}$ & $<1$ & $<1$ & $<1$ & $<1$ & ND & $<1$ & 6 & $<1$ \\
\hline 11 & ND & 929 & NB & 2-Acetyl-1-pyrroline & Popcorn & $\mathrm{O}, \mathrm{RI}, \mathrm{MS}$ & ND & 3 & 4 & 4 & 4 & 2.5 & 6 & 5 \\
\hline 12 & ND & 961 & NB & Dimethyl trisulfide & Cabbage/garlic & $\mathrm{O}, \mathrm{RI}, \mathrm{MS}$ & 2 & 4 & $<1$ & $<1$ & 3 & 2 & 2 & $<1$ \\
\hline 13 & 1306 & 984 & $\mathrm{~A} / \mathrm{NB}$ & 1-Octen-3-one & Mushroom & $\mathrm{O}, \mathrm{RI}, \mathrm{MS}$ & 3 & 3 & $<1$ & 3 & 2 & 2 & 4.5 & 4 \\
\hline 14 & 1234 & 1016 & $\mathrm{~A} / \mathrm{NB}$ & Octanal & Citrus & $\mathrm{O}, \mathrm{RI}, \mathrm{MS}$ & $<1$ & 3 & $<1$ & $<1$ & 2 & 2 & 3 & $<1$ \\
\hline 15 & 1419 & 1030 & $\mathrm{~A} / \mathrm{NB}$ & (E,E)-2,4 heptadienal & Nutty/grainy & $\mathrm{O}, \mathrm{RI}, \mathrm{MS}$ & $<1$ & $<1$ & ND & $<1$ & ND & 2 & $<1$ & ND \\
\hline 16 & ND & 1044 & $\mathrm{NB}$ & 2-Acetyl thiazole & Popcorn & $\mathrm{O}, \mathrm{RI}$ & ND & $<1$ & $<1$ & $<1$ & ND & ND & ND & $<1$ \\
\hline 17 & 1846 & 1052 & $\mathrm{~A} / \mathrm{NB}$ & Benzyl alcohol & Fruity/sweet & $\mathrm{O}, \mathrm{RI}, \mathrm{MS}$ & $<1$ & $<1$ & ND & $<1$ & ND & ND & $<1$ & 2 \\
\hline 18 & ND & 1067 & NB & Gamma terpinene & Pine & $\mathrm{O}, \mathrm{RI}, \mathrm{MS}$ & 2 & $<1$ & ND & $<1$ & ND & ND & ND & $<1$ \\
\hline 19 & 1430 & 1074 & $\mathrm{~A} / \mathrm{NB}$ & 2-Ethyl-3,5-dimethylpyrazine & Smoky/earthy & $\mathrm{O}, \mathrm{RI}$ & $<1$ & $<1$ & ND & $<1$ & $<1$ & $<1$ & $<1$ & $<1$ \\
\hline 20 & ND & 1099 & NB & Sotolon & Sweet & $\mathrm{O}, \mathrm{RI}, \mathrm{MS}$ & ND & ND & ND & ND & 2 & 3 & 3 & ND \\
\hline 21 & ND & 1116 & NB & 2-Methoxy phenol & Smoky/burnt & $\mathrm{O}, \mathrm{RI}, \mathrm{MS}$ & 2 & 3 & $<1$ & $<1$ & 2 & 3 & 3 & 4 \\
\hline 22 & ND & 1123 & NB & Homofuraneol & Sweet & O, RI, MS & 3 & 2 & ND & ND & 2 & 1 & 1 & 3 \\
\hline 23 & 1344 & 1131 & $\mathrm{~A} / \mathrm{NB}$ & Nonanal & Fatty/plastic & $\mathrm{O}, \mathrm{RI}, \mathrm{MS}$ & $<1$ & $<1$ & $<1$ & 3 & 2.5 & $<1$ & ND & 5 \\
\hline 24 & ND & 1157 & NB & 2,4-Dimethylphenol & Mushroom/smoky & $\mathrm{O}, \mathrm{RI}$ & 2 & $<1$ & $<1$ & ND & 2 & 2 & $<1$ & $<1$ \\
\hline 25 & ND & 1165 & $\mathrm{NB}$ & 4-Ethylphenol & Earthy/smoky & $\mathrm{O}, \mathrm{RI}$ & $<1$ & $<1$ & $<1$ & $<1$ & $<1$ & ND & $<1$ & ND \\
\hline 26 & ND & 1193 & NB & Decanal & Carpet/clay & $\mathrm{O}, \mathrm{RI}, \mathrm{MS}$ & $<1$ & 2 & 2 & $<1$ & $<1$ & 2 & 3 & $<1$ \\
\hline 27 & ND & 1212 & NB & (E,Z)-2,4-nonadienal & Cucumber & $\mathrm{O}, \mathrm{RI}, \mathrm{MS}$ & $<1$ & $<1$ & $<1$ & $<1$ & $<1$ & $<1$ & ND & $<1$ \\
\hline 28 & 1968 & 1233 & $\mathrm{~A} / \mathrm{NB}$ & Benzothiazole & Carpet/clay & $\mathrm{O}, \mathrm{RI}$ & $<1$ & 3 & 3 & 3 & 3 & 3 & 3 & 2.5 \\
\hline 29 & 1286 & ND & NB & Unknown & Vomit & $\mathrm{O}$ & ND & ND & 2 & ND & ND & ND & ND & ND \\
\hline 30 & ND & 1350 & NB & 2-Aminoacetophenone & Tortilla & $\mathrm{O}, \mathrm{RI}, \mathrm{MS}$ & 2 & ND & $<1$ & 2 & 3 & 2 & $<1$ & $<1$ \\
\hline 31 & ND & 1362 & NB & (E,E)-2,4 decadienal & Fatty & O, RI, MS & $<1$ & 2 & ND & $<1$ & $<1$ & ND & $<1$ & 2 \\
\hline 32 & 1444 & ND & A & Acetic acid & Vinegar/sour & $\mathrm{O}, \mathrm{RI}$ & $<1$ & 3 & 2.5 & 4 & 3 & $<1$ & ND & 3 \\
\hline 33 & ND & 1466 & NB & Beta ionone & Sweet/fatty & $\mathrm{O}, \mathrm{RI}, \mathrm{MS}$ & 2 & $<1$ & $<1$ & 2 & $<1$ & 3 & $<1$ & 2 \\
\hline 34 & 1514 & ND & A & Propionic acid & Garbage & $\mathrm{O}, \mathrm{RI}$ & 2 & 3 & $<1$ & ND & 3 & $<1$ & 2 & ND \\
\hline 35 & ND & 1543 & NB & Unknown & Licorice/hay & $\mathrm{O}$ & ND & ND & 3 & ND & $<1$ & ND & $<1$ & 3 \\
\hline 36 & 1551 & ND & $\mathrm{A}$ & 5-Methyl furfural & Smoky & $\mathrm{O}, \mathrm{RI}$ & $<1$ & 2 & $<1$ & $<1$ & ND & ND & ND & ND \\
\hline 37 & ND & 1628 & NB & Unknown & Stale/dough & $\mathrm{O}$ & $<1$ & $<1$ & $<1$ & 2 & 3 & 3 & $<1$ & 2.5 \\
\hline 38 & 1684 & ND & $\mathrm{A}$ & Butanoic acid & Vomit & $\mathrm{O}, \mathrm{RI}, \mathrm{MS}$ & 2.5 & 3 & 3 & 4 & 3 & ND & 4 & 5 \\
\hline 39 & 1764 & ND & A & Pentanoic acid & Smoky/stinky feet & $\mathrm{O}, \mathrm{RI}, \mathrm{MS}$ & 2 & 3 & ND & ND & 2 & $<1$ & ND & 3 \\
\hline 40 & ND & 1784 & NB & Delta-dodecalactone & Sweet/coconut & $\mathrm{O}, \mathrm{RI}$ & $<1$ & $<1$ & $<1$ & $<1$ & 3 & 2 & 2 & $<1$ \\
\hline 41 & 1809 & ND & $\mathrm{A}$ & Hexanoic acid & FFA/cheesy & $\mathrm{O}, \mathrm{RI}, \mathrm{MS}$ & $<1$ & $<1$ & 3 & $<1$ & 3 & $<1$ & 3 & ND \\
\hline 42 & ND & 2092 & NB & 1-Octadecanol & Soap & $\mathrm{O}, \mathrm{RI}$ & 2 & 3 & 2 & ND & ND & $<1$ & $<1$ & $<1$ \\
\hline 43 & $>2,100$ & ND & A & Decanoic acid & Soapy & $\mathrm{O}, \mathrm{RI}, \mathrm{MS}$ & ND & $<1$ & ND & ND & ND & $<1$ & ND & 2 \\
\hline
\end{tabular}

Retention indices (RI) from gas chromatography-olfactometry data on the WAX and ZB-5 columns.

${ }^{2} \mathrm{~A}=$ acid fraction, $\mathrm{NB}=$ neutral/basic fraction from solvent extraction with gas chromatography-olfactometry.

${ }^{3}$ Odor description at gas chromatography sniffing port.

${ }^{4} \mathrm{O}=$ olfactometry, and MS = mass spectrometry.

${ }^{5} \mathrm{ND}=$ not detected. 
butanoic acid. Aroma active compounds with high $\log _{3}$ FD factors $(>2)$ in MPC that were not high in MPI or MCC were dimethyl disulfide, hexanal, 1-hexen-3-one, heptanal, 1-octen-3-one, octanal, homofuraneol, decanal, and pentanoic acid. Aroma active compounds with high $\log _{3}$ FD factors in MPI that were not in MPC or MCC were dimethyl trisulfide, 2-aminoacetophenone, propionic acid, and delta-dodecalactone. The MCC had relatively few aroma active compounds with high $\log _{3}$ FD factors, and only $\beta$-ionone had a high $\log _{3}$ FD factor in MCC and not in MPC or MPI.

Sensory profiles of the casein, MPC, and MCC samples varied $(P<0.05$; Table 4$)$. Tortilla/corn chip and cardboard flavors were predominant in all samples, with the exception of acid casein, which was highest in tortilla/corn chip flavor followed by brothy/animal flavor. Only acid and rennet caseins exhibited animal flavor. Tortilla/corn chip flavor has previously been identified as a primary flavor of rennet casein and MPC 80/MPI by descriptive analysis (Karagul-Yuceer et al., 2003; Drake et al., 2009). The flavor of caseins has been characterized by a variety of descriptors including ani$\mathrm{mal} /$ wet dog, cooked, potato/brothy, sulfur, glue, tortilla, and cardboard (Karagul-Yuceer et al., 2003; Drake et al., 2009). Karagul-Yuceer et al. (2003) identified the most potent odorant in rennet casein (based on $\log _{3} \mathrm{FD}$ factors) as 2-aminoacetophenone, which contributed a tortilla and grapelike odor. This compound was previously reported to contribute to a glue/burnt feathers flavor in stored casein (Ramshaw and Dunstone, 1969), stored dry milk (Parks et al., 1964), and sterilized concentrated milk (Arnold et al., 1966). Despite its high aroma activity, Karagul-Yuceer et al. (2003) concluded that the typical odor of rennet casein was primarily caused by hexanoic acid, indole, guaiacol, and p-cresol based upon odor activity values (OAV) and sensory analysis of model mixtures of flavor compounds. In the current study, the significance of 2-aminoacetophenone is consistent with past studies.

Milk proteins (MPC and MPI) have been characterized by descriptors including cooked/milky, sweet aromatic, tortilla, potato/brothy, cardboard, and animal (Drake et al., 2009). Drake et al. (2009) also reported that MPC with lower protein content $(56 \%$ protein by dry weight) were characterized by fluid milk types of flavor (cooked/milky, sweet aromatic, sweet taste, and cereal, and as protein content increased, the flavor profiles changed and MPC70, MPC 80, and MPI were characterized by tortilla, brothy, cardboard, and animal flavors and higher astringency. The current study characterized milk proteins using the same descriptors, and the MPC 70 and 80 were characterized by low but distinct intensity of tortilla and cardboard flavor, cooked/milky, and sweet aromatic flavors (Table 4).

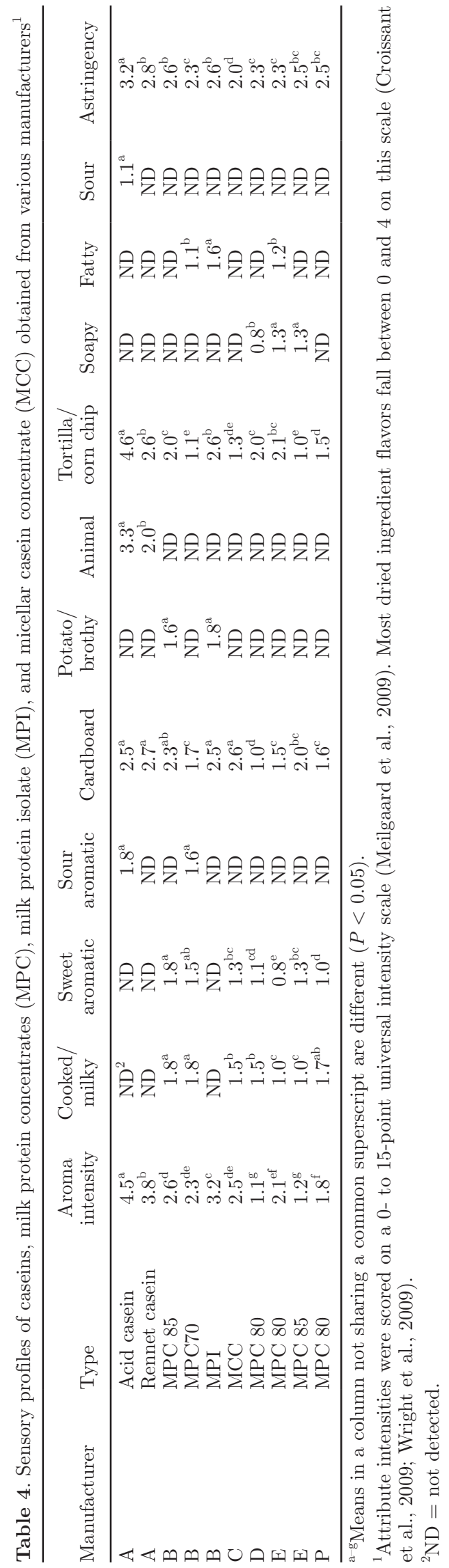

Journal of Dairy Science Vol. 99 No. 6, 2016 
Seven odor active volatile compounds (2-acetylpyrroline, 1-octen-3-one, 2-aminoacetophenone, nonanal, heptanal, DMTS, and methional) from the neutral/ basic portion of SAFE extracts were quantified by standard curves. Nine volatile fatty acids (butyric, pentanoic, hexanoic, heptanoic, octanoic, nonanoic, decanoic, dodecanoic, and tetradecanoic) from the acid portion of the SAFE extracts were quantified by standard curves. Three other odor active volatile compounds (hexanal, 1-hexen-3-one, and DMDS) were quantified via SPME. Relative abundance of 15 other volatile compounds was determined by SPME headspace GC-MS (Table 4). Sensory profiles of milk proteins and caseins were generally consistent with volatile compound concentrations and relative abundance (Tables 4 and 5). A correlation between concentration of 2-aminoacetophenone and tortilla/corn chip flavor was observed (Figure 1, $\left.\mathrm{R}^{2}=0.87, P<0.05\right)$. The importance of 2 -aminoacetophenone to tortilla/corn chip flavor is well established in both corn tortillas and taco shells (Karahadian and Johnson, 1993; Buttery et al., 1994; Buttery and Ling, 1995, 1998) and casein (Badings, 1991; Karagul-Yuceer et al., 2003). The current study confirms the importance of 2-aminoacetophenone to the tortilla flavor of dried milk proteins. However, tortilla flavor in milk proteins may also be caused by several volatile compounds and not just 2-aminoacetophenone based on previous studies (Karagul-Yuceer et al., 2003) and on aroma active compounds detected in the current study. Many of the odor active compounds in corn tortillas, chips, and taco shells have also been identified in the milk protein products of the current study, including E,E and E,Z 2,4 decadienal, acetic acid, 3-methylbutanal, 2-acetyl1-pyrroline, 2-aminoacetophenone, E-2-nonenal, 2 -methylbutanal, E-2-decenal, methional, $\beta$-ionone, hexanal, 1-octen-3-ol, and 2-ethyl-3,5-dimethylpyrazine (Tables 2 and 3; Buttery and Ling, 1995, 1998).

Acid casein was characterized by high aroma intensity, sour aromatic, cardboard, animal flavor, and tortilla/corn chip flavors. Acid casein also had high concentrations of 2-aminoacetophenone, E-2-heptenal, and E-2-nonenal. Rennet casein was also characterized by high aroma intensity, cardboard, and animal flavors, but was high in phenylacetaldehyde, sotolon, E-2-heptenal, 2,4 decadienal, 1-hexen-3-one, hexanal, DMDS, DMTS, and 2-pentyl furan. Despite similar sensory profiles, compound concentrations and relative abundance were highly variable likely due to distinct differences in manufacture and processing. It is interesting to note that hexanoic acid was identified as an important flavor contributor to rennet casein in past studies (KaragulYuceer et al., 2003), but hexanoic acid was not a factor in the flavor of rennet casein in the current study. This result is consistent with the SAFE GC-O data, which also lacked a hexanoic acid aroma event. The effects of raw materials, processing and storage all affect flavor and flavor chemistry of dairy ingredients (Smith et al., 2016). It is likely that this discrepancy is due to one or several those factors. Volatile compound differences were also observed among the MPC 80 and MPI from different suppliers.

Many of the volatile compounds related to flavor in milk proteins are either formed by lipid oxidation or Maillard browning. The production of 2-aminoacetophenone in corn tortilla/chip products occurs when corn is heated and exposed to an alkali (Buttery and Ling, 1998), but past studies have also suggested that alkaline conditions catalyzed air oxidation of the amino acid tryptophan to the compound kynurenine, which is then degraded to 2-aminoacetophenone (Tabone et al., 1951). It is possible that in milk protein powders, 2 -aminoacetophenone is formed by the oxidation of tryptophan in solution. However, this seems unlikely due to the lack of a strong oxidative step during the manufacture of milk proteins necessary to catalyze the oxidation of tryptophan. It also seems unlikely due to the lack of 2-aminoacetophenone content, and tortilla off-flavor in whey protein concentrates, which often undergo oxidative bleaching during processing (Croissant et al., 2009; Jervis et al., 2012; Fox et al., 2013). The tryptophan content of $\beta$-lactoglobulin and $\alpha$-lactalbumin (whey proteins) is much higher than that of $\alpha$ - and $\beta$-caseins (Spies, 1967), which would suggest that if the alkali breakdown of tryptophan was the primary source of 2-aminoacetophenone in milk proteins, it would most certainly also be a major flavor component in whey protein concentrates. Past studies (Spacek, 1954; Parks et al., 1967) have demonstrated that alkaline degradation of kynurenine results in a $>90 \%$ yield of 2 -aminoacetophenone, whereas the yield of 2-aminoacetophenone from tryptophan by alkaline degradation is less than $0.1 \%$. Parks et al. (1964) also determined that kynurenine occurred naturally in bovine milk. It is possible that the 2 -aminoacetophenone concentration in milk and milk protein powders is due to the free kynurenine in milk, which may be broken down by nonalkaline means or remains in the cheese during the cheese-make procedure of liquid whey manufacture, preventing its presence in whey protein.

Alkanals and alkenals, such as decadienal, methylbutanal, nonenal, decenal, and hexanal, are typical products of lipid oxidation. Increased lipid oxidation products over processing, storage, or both is expected and has been demonstrated in pasteurized fluid milk, SMP, whole milk powder, and dried whey protein ingredients (Karagul-Yuceer et al., 2002; Karatapanis et al., 2006; Lloyd et al., 2009; Wright et al., 2009). The autoxidative mechanisms of unsaturated fatty acids in the milk 
Table 5. Mean concentration or relative abundance of selected compounds in milk protein and casein powders ${ }^{1}$

\begin{tabular}{|c|c|c|c|c|c|c|c|c|c|}
\hline Item & A: Acid casein & A: Rennet casein & B: MPC 85 & B: MPI & $\mathrm{C}: \mathrm{MCC}$ & D: MPC 80 & E: MPC 80 & E: MPC 85 & P: MPC 80 \\
\hline \multicolumn{10}{|l|}{$\overline{\text { Concentration }^{2}(\mu \mathrm{g} / \mathrm{kg})}$} \\
\hline 2-Acetylpyrroline & $0.011^{\mathrm{d}}$ & $1.88^{\mathrm{b}}$ & $0.502^{\mathrm{d}}$ & $0.011^{\mathrm{d}}$ & $3.73^{\mathrm{a}}$ & $1.14^{\mathrm{c}}$ & $0.171^{\mathrm{d}}$ & $0.523^{\mathrm{d}}$ & $1.07^{\mathrm{c}}$ \\
\hline 1-Hexen-3-one* & $9.70^{\mathrm{b}}$ & $264^{\mathrm{a}}$ & $2.35^{\mathrm{b}}$ & $0.451^{\mathrm{b}}$ & $0.315^{\mathrm{b}}$ & $1.20^{\mathrm{b}}$ & $1.00^{\mathrm{b}}$ & $0.393^{\mathrm{b}}$ & $1.11^{\mathrm{b}}$ \\
\hline 1-Octen-3-one & $0.238^{\mathrm{b}}$ & $104^{\mathrm{b}}$ & $528^{\mathrm{a}}$ & $20.3^{\mathrm{b}}$ & $9.16^{\mathrm{b}}$ & $4.98^{\mathrm{b}}$ & $20.1^{\mathrm{b}}$ & $52.8^{\mathrm{b}}$ & $50.7^{\mathrm{b}}$ \\
\hline 2-Aminoacetophenone & $12.7^{\mathrm{a}}$ & $1.01^{\mathrm{b}}$ & $0.053^{\mathrm{e}}$ & $0.060^{\mathrm{e}}$ & $0.076^{\mathrm{e}}$ & $0.630^{\mathrm{c}}$ & $0.102^{\mathrm{de}}$ & $0.055^{\mathrm{e}}$ & $0.238^{\mathrm{d}}$ \\
\hline Hexanal* & $52.8^{\mathrm{b}}$ & $217^{\mathrm{a}}$ & $27.3^{\mathrm{bc}}$ & $2.31^{\mathrm{c}}$ & $1.57^{\mathrm{c}}$ & $4.95^{\mathrm{c}}$ & $5.29^{\mathrm{c}}$ & $7.96^{\mathrm{c}}$ & $4.53^{\mathrm{c}}$ \\
\hline Heptanal & $1.16^{\mathrm{b}}$ & $210^{\mathrm{a}}$ & $190^{\mathrm{a}}$ & $3.50^{\mathrm{b}}$ & $18.8^{\mathrm{b}}$ & $57.9^{\mathrm{b}}$ & $203^{\mathrm{a}}$ & $30.8^{\mathrm{b}}$ & $63.4^{\mathrm{b}}$ \\
\hline Nonanal & $20.5^{\mathrm{e}}$ & $16.3^{\mathrm{e}}$ & $644^{\mathrm{a}}$ & $76.8^{\text {cde }}$ & $167^{\text {bcd }}$ & $210^{\mathrm{bc}}$ & $279^{\mathrm{b}}$ & $690^{\mathrm{a}}$ & $64.0^{\mathrm{de}}$ \\
\hline DMDS* & $6.27^{\mathrm{b}}$ & $26.2^{\mathrm{a}}$ & $0.500^{\mathrm{b}}$ & $0.057^{\mathrm{b}}$ & $0.062^{\mathrm{b}}$ & $0.093^{\mathrm{b}}$ & $0.105^{\mathrm{b}}$ & $0.058^{\mathrm{b}}$ & $0.460^{\mathrm{b}}$ \\
\hline DMTS & $0.092^{\mathrm{b}}$ & $36.7^{\mathrm{a}}$ & $2.50^{\mathrm{b}}$ & $0.293^{\mathrm{b}}$ & $0.593^{\mathrm{b}}$ & $0.455^{\mathrm{b}}$ & $1.18^{\mathrm{b}}$ & $4.48^{\mathrm{b}}$ & $3.89^{\mathrm{b}}$ \\
\hline Methional & $0.082^{\mathrm{d}}$ & $18.2^{\mathrm{b}}$ & $18.9^{\mathrm{b}}$ & $0.095^{\mathrm{d}}$ & $2.66^{\mathrm{cd}}$ & $36.1^{\mathrm{a}}$ & $8.22^{\mathrm{c}}$ & $0.108^{\mathrm{d}}$ & $1.99^{\mathrm{d}}$ \\
\hline \multicolumn{10}{|l|}{ Concentration $^{3}(\mathrm{mg} / \mathrm{kg})$} \\
\hline Butyric & $6.73^{\mathrm{b}}$ & $6.15^{\mathrm{b}}$ & $3.05^{\mathrm{b}}$ & $11.2^{\mathrm{b}}$ & $0.973^{\mathrm{b}}$ & $9.10^{\mathrm{b}}$ & $109^{\mathrm{a}}$ & ND & $2.48^{\mathrm{b}}$ \\
\hline Pentanoic & $0.083^{\mathrm{c}}$ & $38.6^{\mathrm{ab}}$ & ND & $3.03^{\mathrm{c}}$ & $4.59^{\mathrm{bc}}$ & $25.3^{\mathrm{abc}}$ & $3.43^{\mathrm{bc}}$ & $2.48^{\mathrm{c}}$ & $58.2^{\mathrm{a}}$ \\
\hline Hexanoic & $5.42^{\mathrm{b}}$ & $0.015^{\mathrm{b}}$ & $5.06^{\mathrm{b}}$ & $9.18^{\mathrm{b}}$ & $0.012^{\mathrm{b}}$ & $0.209^{\mathrm{b}}$ & $62.6^{\mathrm{a}}$ & $45.5^{\mathrm{a}}$ & $10.7^{\mathrm{b}}$ \\
\hline Heptanoic & $0.022^{\mathrm{c}}$ & $0.663^{\text {bc }}$ & ND & $0.710^{\mathrm{bc}}$ & $1.45^{\mathrm{bc}}$ & $35.3^{\mathrm{ab}}$ & $2.34^{\mathrm{bc}}$ & $2.09^{\mathrm{bc}}$ & $65.8^{\mathrm{a}}$ \\
\hline Octanoic & $1.89^{\mathrm{b}}$ & $0.465^{\mathrm{b}}$ & $7.43^{\mathrm{b}}$ & $27.6^{\mathrm{b}}$ & $10.0^{\mathrm{b}}$ & ND & $29.1^{\mathrm{b}}$ & $107^{\mathrm{a}}$ & $108^{\mathrm{a}}$ \\
\hline Nonanoic & $0.035^{\mathrm{b}}$ & ND & ND & $7.25^{\mathrm{ab}}$ & $6.58^{\mathrm{ab}}$ & $17.9^{\mathrm{ab}}$ & $4.18^{\mathrm{ab}}$ & $9.49^{\mathrm{ab}}$ & $41.8^{\mathrm{a}}$ \\
\hline Decanoic & $0.044^{\mathrm{c}}$ & $0.078^{\mathrm{c}}$ & $0.259^{\mathrm{c}}$ & $20.5^{\mathrm{b}}$ & $0.005^{\mathrm{c}}$ & $2.71^{\mathrm{c}}$ & $10.0^{\mathrm{bc}}$ & $43.6^{\mathrm{a}}$ & $35.2^{\mathrm{a}}$ \\
\hline Dodecanoic & $2.70^{\mathrm{c}}$ & $0.234^{\mathrm{c}}$ & $0.020^{\mathrm{c}}$ & $19.7^{\mathrm{bc}}$ & $0.005^{\mathrm{c}}$ & $111^{\mathrm{ab}}$ & $19.1^{\mathrm{bc}}$ & $100^{\mathrm{ab}}$ & $139^{\mathrm{a}}$ \\
\hline Tetradecanoic & $4.64^{\mathrm{b}}$ & $0.312^{\mathrm{b}}$ & $0.023^{\mathrm{b}}$ & $2.09^{\mathrm{b}}$ & $0.007^{\mathrm{b}}$ & $0.038^{\mathrm{b}}$ & $1.77^{\mathrm{b}}$ & $21.4^{\mathrm{ab}}$ & $61.7^{\mathrm{a}}$ \\
\hline \multicolumn{10}{|c|}{ Relative abundance $(\mu \mathrm{g} / \mathrm{kg})$} \\
\hline Phenylacetaldehyde & $2.28^{\mathrm{b}}$ & $31.3^{\mathrm{a}}$ & $0.089^{\mathrm{b}}$ & $4.81^{\mathrm{b}}$ & $0.184^{\mathrm{b}}$ & $1.56^{\mathrm{b}}$ & $43.4^{\mathrm{a}}$ & $8.32^{\mathrm{b}}$ & $0.167^{\mathrm{b}}$ \\
\hline Sotolon & $0.060^{\mathrm{b}}$ & $87.5^{\mathrm{a}}$ & $0.199^{\mathrm{b}}$ & $0.678^{\mathrm{b}}$ & $0.071^{\mathrm{b}}$ & $0.150^{\mathrm{b}}$ & $71.2^{\mathrm{a}}$ & $18.5^{\mathrm{b}}$ & $5.06^{\mathrm{b}}$ \\
\hline Undecanal & $11.1^{\mathrm{cd}}$ & $7.47^{\mathrm{cd}}$ & $21.3^{\mathrm{bc}}$ & $111^{\mathrm{a}}$ & $4.32^{\mathrm{d}}$ & $34.3^{\mathrm{b}}$ & $17.5^{\mathrm{cd}}$ & $5.20^{\mathrm{d}}$ & $7.56^{\mathrm{cd}}$ \\
\hline E,Z 2,6 Nonandienal & $2.00^{\mathrm{c}}$ & $2.50^{\mathrm{c}}$ & $0.302^{\mathrm{c}}$ & $26.9^{\mathrm{a}}$ & $0.143^{\mathrm{c}}$ & $1.86^{\mathrm{c}}$ & $13.2^{\mathrm{b}}$ & $0.561^{\mathrm{c}}$ & $0.765^{\mathrm{c}}$ \\
\hline$\gamma$-Terpinene & $3.58^{\mathrm{b}}$ & $0.109^{\mathrm{c}}$ & $0.062^{\mathrm{c}}$ & $15.0^{\mathrm{a}}$ & $0.564^{\mathrm{c}}$ & $0.087^{\mathrm{c}}$ & $0.424^{\mathrm{c}}$ & $2.38^{\mathrm{b}}$ & $0.127^{\mathrm{c}}$ \\
\hline E-2-Heptenal & $13.3^{\mathrm{ab}}$ & $25.2^{\mathrm{a}}$ & $0.170^{\mathrm{b}}$ & $3.78^{\mathrm{b}}$ & $5.93^{\mathrm{b}}$ & $3.70^{\mathrm{b}}$ & $3.71^{\mathrm{b}}$ & $15.6^{\mathrm{ab}}$ & $0.611^{\mathrm{b}}$ \\
\hline$\gamma$-Dodecalactone & $4.88^{\mathrm{b}}$ & $1.08^{\mathrm{b}}$ & $1.58^{\mathrm{b}}$ & $69.2^{\mathrm{a}}$ & $8.75^{\mathrm{b}}$ & $2.14^{\mathrm{b}}$ & $8.70^{\mathrm{b}}$ & $1.86^{\mathrm{b}}$ & $2.73^{\mathrm{b}}$ \\
\hline Guaiacol & $10.3^{\mathrm{c}}$ & $8.67^{\mathrm{cd}}$ & $0.142^{\text {de }}$ & $21.8^{\mathrm{b}}$ & $0.079^{\mathrm{e}}$ & $0.363^{\text {de }}$ & $98.0^{\mathrm{a}}$ & $4.66^{\text {cde }}$ & $0.360^{\text {de }}$ \\
\hline 2,4 Decadienal & $0.158^{\mathrm{b}}$ & $40.5^{\mathrm{a}}$ & $0.169^{\mathrm{b}}$ & $2.28^{\mathrm{b}}$ & $2.75^{\mathrm{b}}$ & $0.717^{\mathrm{b}}$ & $11.7^{\mathrm{b}}$ & $18.5^{\mathrm{b}}$ & $4.31^{\mathrm{b}}$ \\
\hline Octanal & $0.881^{\mathrm{d}}$ & $17.0^{\mathrm{b}}$ & $8.89^{\mathrm{bcd}}$ & $2.75^{\mathrm{cd}}$ & $6.09^{\mathrm{cd}}$ & $2.01^{\mathrm{cd}}$ & $4.67^{\mathrm{cd}}$ & $10.6^{\mathrm{bc}}$ & $83.5^{\mathrm{a}}$ \\
\hline E 2-Nonenal & $197^{\mathrm{a}}$ & $6.47^{\mathrm{bcd}}$ & $0.060^{\mathrm{d}}$ & $3.26^{\text {cd }}$ & $0.082^{\mathrm{d}}$ & $25.4^{\mathrm{b}}$ & $2.89^{\mathrm{d}}$ & $5.78^{\text {bcd }}$ & $25.1^{\mathrm{bc}}$ \\
\hline 2-Pentylfuran & $2.25^{\mathrm{b}}$ & $35.2^{\mathrm{a}}$ & $0.202^{\mathrm{b}}$ & $24.8^{\mathrm{a}}$ & $2.30^{\mathrm{b}}$ & $0.562^{\mathrm{b}}$ & $36.0^{\mathrm{a}}$ & $3.35^{\mathrm{b}}$ & $4.08^{\mathrm{b}}$ \\
\hline E 2-Decenal & $6.33^{\mathrm{e}}$ & $22.6^{\mathrm{cd}}$ & $0.341^{\mathrm{e}}$ & $1.05^{\mathrm{e}}$ & $0.275^{\mathrm{e}}$ & $12.8^{\mathrm{de}}$ & $56.5^{\mathrm{b}}$ & $26.8^{\mathrm{c}}$ & $75.3^{\mathrm{a}}$ \\
\hline Decanal & $0.536^{\mathrm{d}}$ & $10.7^{\mathrm{c}}$ & $7.10^{\mathrm{cd}}$ & $2.48^{\mathrm{d}}$ & $5.84^{\mathrm{cd}}$ & $2.39^{\mathrm{d}}$ & $48.3^{\mathrm{a}}$ & $7.54^{\mathrm{cd}}$ & $21.7^{\mathrm{b}}$ \\
\hline E,E 2,4 Heptadienal & $2.36^{\text {cde }}$ & $5.60^{\mathrm{c}}$ & $0.168^{\mathrm{e}}$ & $0.749^{\mathrm{de}}$ & $3.10^{\text {cde }}$ & $0.589^{\mathrm{de}}$ & $30.5^{\mathrm{b}}$ & $4.70^{\mathrm{cd}}$ & $39.7^{\mathrm{a}}$ \\
\hline
\end{tabular}

( ${ }^{\mathrm{a}} \mathrm{Means}$ in a row not sharing a common superscript are different $(P<0.05)$

${ }^{1} \mathrm{MPC}=$ milk protein concentrate; MPI = milk protein isolate; $\mathrm{MCC}=$ micellar casein concentrate. DMDS = dimethyl disulfide; DMTS $=$ dimethyl trisulfide.

${ }^{2}$ Compounds from neutral/basic portion of solvent-assisted flavor extraction (SAFE) unless otherwise indicated [some compounds were extracted by solid-phase microextraction $\lesssim \quad$ (SPME) headspace, not SAFE, as denoted by an *].

๑ ${ }^{3}$ Compounds from acid portion of SAFE. 


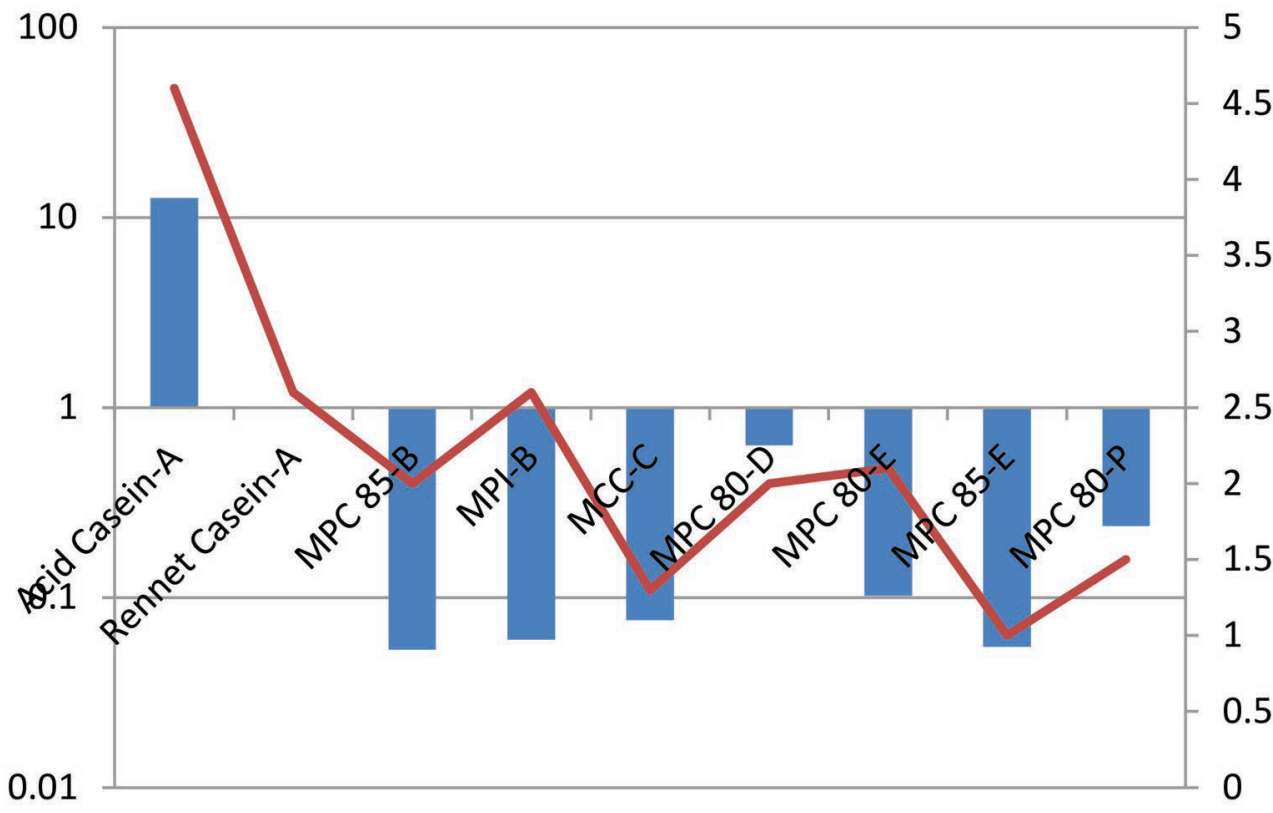

4.5

3.5

3

2.5

2-aminoacetophenone

Tortilla/corn chip flavor

Figure 1. Concentration ( $\mu \mathrm{g} / \mathrm{kg}$; left y-axis) of 2-aminoacetophenone compared with tortilla/corn chip flavor intensity (right y-axis) in select milk proteins and caseins. Color version available online.

lead to the formation of many aldehydes. They have low flavor thresholds and are commonly characterized by oily, fatty, or tallow odors (Azzara and Campbell, 1992). Whitson et al. (2010) demonstrated that a combination of lipid oxidation products and DMTS caused cardboard flavor in whey proteins. In certain concentrations and combinations with 2-aminoacetophenone, some or all of these compounds may also contribute to tortilla flavor in milk proteins. The alcohol 1-octen-3-ol was important to both the flavor of the milk proteins in this study and corn tortillas and corn chips (Buttery and Ling, 1995, 1998).

The compound 2-acetyl-1-pyroline is characterized by a popcorn aroma and is a result of the reaction between the AA proline or ornithine and the reactive sugar-degradation product 2-oxopropanal (Schieberle, 1995). The compound 2-acetyl-1-pyroline is commonly found in many fresh dairy ingredients such as SMP (Karagul-Yuceer and Cadwallader, 2001) and fluid and dried whey powder (Karagul-Yuceer et al., 2003; Mahajan et al., 2004) and contributes a popcorn and cooked flavor. This compound is attributed to sweet aromatic and cooked/milky flavors in milk proteins.

Methional has an aroma similar to boiled potatoes. Methional formation is a result of Strecker degradation of methionine (Tressl et al., 1989) or is a result of methionine breakdown when milk is exposed to light (Badings, 1991). It is also a common flavor-active component of dairy protein powders including liquid whey (Karagul-Yuceer et al., 2003), whey protein concentrate and whey protein isolate (Carunchia Whetstine et al., 2005), milk powders (Karagul-Yuceer and Cadwallader, 2001), and rennet casein (Karagul-Yuceer et al., 2003). The compound 2-ethyl-3,5-dimethylpyrazine is also a Maillard browning product, specifically produced by Strecker degradation (Manley and Fagerson, 1970) and has a roasted earthy aroma (Grosch and Schieberle, 1997). Products formed from Strecker degradation are heat induced and would likely have higher concentrations in products with high heat treatments.

\section{Experiment 2}

Furosine Analysis of MPC During Storage. As expected, furosine levels increased in MPC 45 and 80 over time (Figure 2). Protein level, storage time and storage temperature had an effect on furosine production $(P<0.05)$. The MPC 45 at $3^{\circ} \mathrm{C}$ demonstrated a $31 \%$ furosine increase over $12 \mathrm{mo}$, whereas the MPC 45 at $25^{\circ} \mathrm{C}$ and $40^{\circ} \mathrm{C}$ demonstrated a 650 and $980 \%$ increase, respectively (Figure 2). The MPC 80 at $3^{\circ} \mathrm{C}$ demonstrated an $82 \%$ furosine increase over 12 mo, whereas the MPC 45 at 25 and $40^{\circ} \mathrm{C}$ demonstrated a 510 and $820 \%$ increase, respectively (Figure 2). Storage temperature had a larger effect on furosine concentration than protein level.

Furosine is a commonly used measure of protein-bound Amadori products and therefore measures the extent of the Maillard reaction in dairy proteins (Erbersdobler, 1986). Reduction of solubility in milk proteins could 


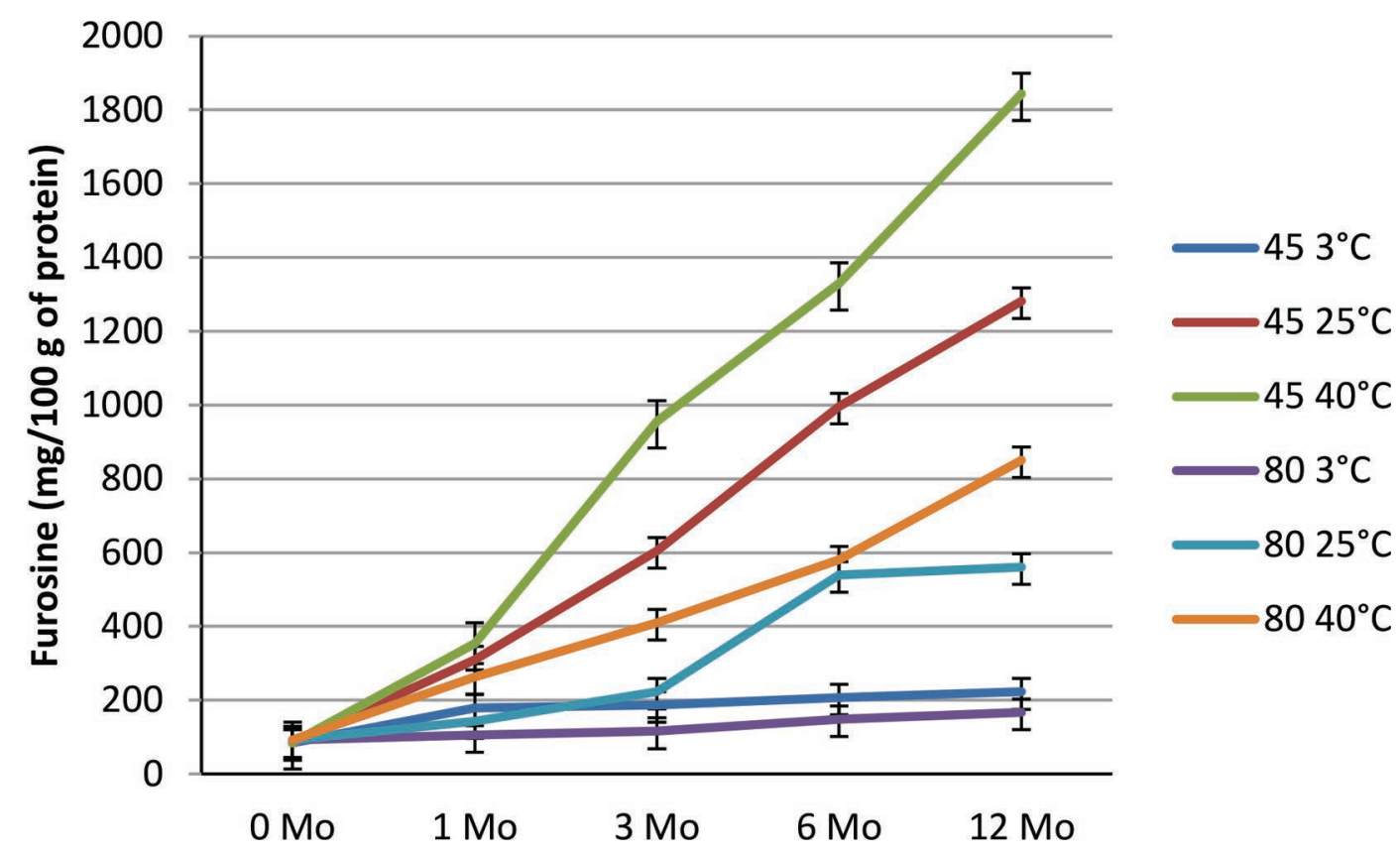

Figure 2. Furosine concentration (mg/100 $\mathrm{g}$ of protein) in milk protein concentrate (MPC) 45 and 80 stored at 3,25 , and $40^{\circ} \mathrm{C}$ for $1,3,6$, and 12 mo. Color version available online.

be due to covalent cross-linking of the proteins, hydrophobic or hydrogen bonds (Anema et al., 2006; Havea, 2006). The Maillard reaction may not be the primary cause of solubility loss in milk proteins, but Maillard browning products such as glyoxal or methylglyoxal can react with lysine or arginine, causing cross-linking of proteins and subsequent loss of solubility (Lederer and Klaiber, 1999; Biemel et al., 2001; Le et al., 2011b, 2013). Maillard reactions play an important role in the shelf-life of milk proteins, and furosine is a useful measurement to compare the degree of Maillard browning that has taken place (Le et al., 2011a). The MPC 45

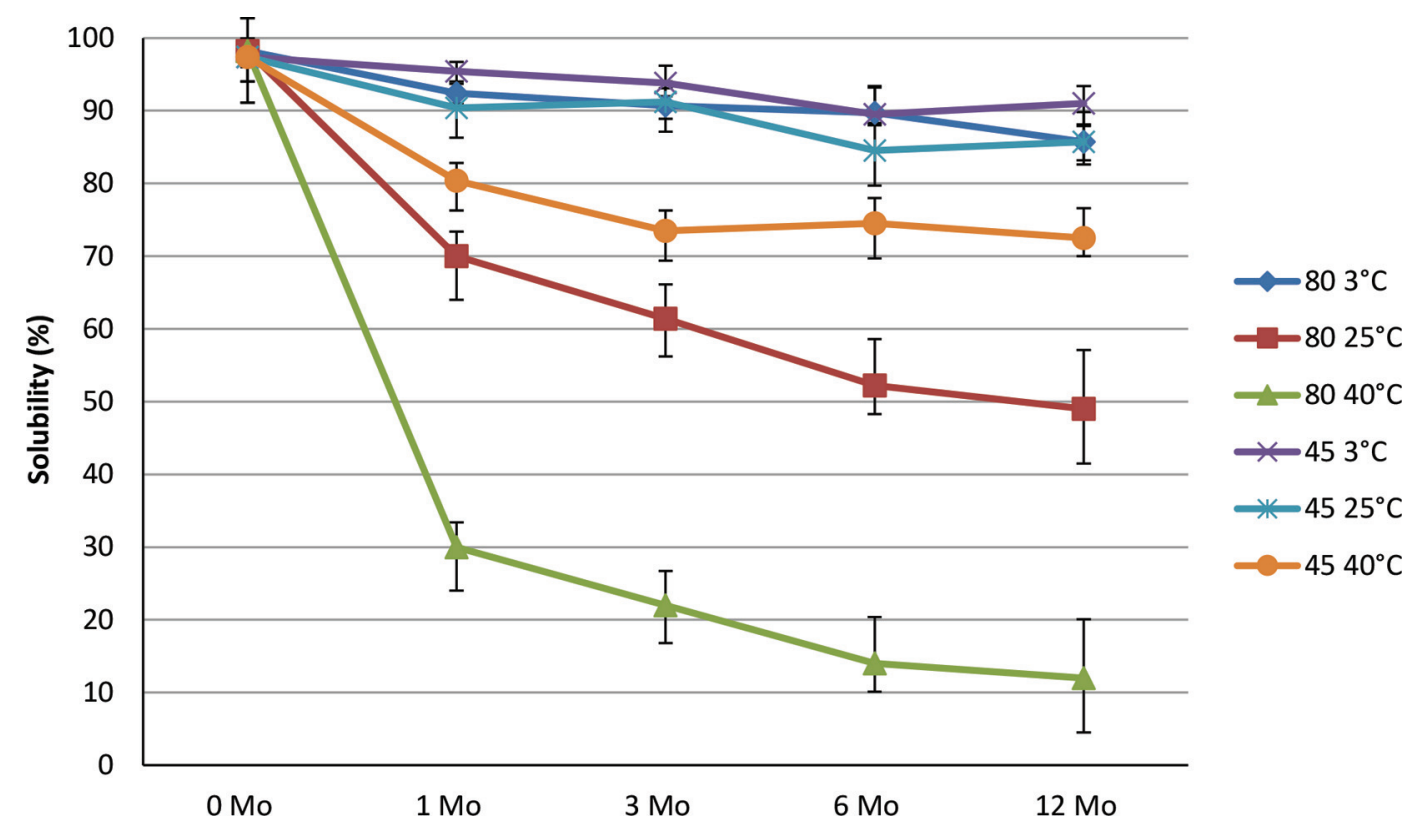

Figure 3. Percent solubility of milk protein concentrate (MPC) 45 and 80 stored at 3,25 , and $40^{\circ} \mathrm{C}$ for $0,1,3,6$, and 12 mo. Color version available online. 
$\underset{\varnothing}{\subseteq} \quad$ Table 6. Mean relative abundance $(\mu \mathrm{g} / \mathrm{kg})$ of select volatile compounds by protein concentration, storage time, and storage temperature ${ }^{1}$

Pentanal Hexanal 2-Pentylfuran Octanal

DMS DMDS DMTS Heptanal Methional Nonanal 2 MB

3 MB 1-Octen3one 2,4 Decadiena

\begin{tabular}{|c|c|c|c|c|c|c|c|c|c|c|c|c|c|c|}
\hline $45 \times \mathrm{T} 1 \times 3 \mathrm{C}$ & $1.26^{\text {efghi }}$ & $3.47^{\mathrm{k}}$ & $0.312^{\mathrm{c}}$ & $318^{\mathrm{kl}}$ & $230^{\text {cdef }}$ & $152^{\mathrm{g}}$ & $0.052^{\mathrm{de}}$ & $0.323^{\mathrm{h}}$ & $0.084^{\text {efg }}$ & $0224^{\mathrm{d}}$ & $153^{\text {ghi }}$ & $0432^{\text {defg }}$ & $111^{\mathrm{ef}}$ & $0268^{\mathrm{g}}$ \\
\hline $45 \times \mathrm{T} 1 \times 25 \mathrm{C}$ & $1.32^{\text {efgh }}$ & $3.74^{\mathrm{k}}$ & $0.297^{\mathrm{c}}$ & $35.2^{\mathrm{jkl}}$ & $2.41^{\text {bcdef }}$ & $4.31^{\mathrm{g}}$ & $0.059^{\text {cde }}$ & $0.396^{\text {gh }}$ & $0.073^{\text {efg }}$ & $0.236^{\mathrm{d}}$ & $1.56^{\text {gh }}$ & $0.523^{\text {bcde }}$ & $1.35^{\operatorname{def}}$ & $0.554^{\mathrm{fg}}$ \\
\hline $45 \times \mathrm{T} 1 \times 40 \mathrm{C}$ & $1.38^{\mathrm{efg}}$ & $5.24^{\mathrm{jk}}$ & $0.294^{\mathrm{c}}$ & $31.0^{1}$ & $2.64^{\text {abcde }}$ & $15.9^{\mathrm{fg}}$ & $0.050^{\mathrm{de}}$ & $0.346^{\mathrm{h}}$ & $0.079^{\mathrm{efg}}$ & $0.196^{\mathrm{d}}$ & $1.75^{\mathrm{fg}}$ & $0.088^{\mathrm{kl}}$ & $1.29^{\mathrm{def}}$ & $0.556^{\mathrm{fg}}$ \\
\hline $45 \times \mathrm{T} 3 \times 3 \mathrm{C}$ & $1.14^{\text {fghij }}$ & $4.57^{\mathrm{jk}}$ & $0.309^{\mathrm{c}}$ & $48.1^{\text {hijkl }}$ & $1.83^{\mathrm{f}}$ & $3.16^{\mathrm{g}}$ & $0.052^{\mathrm{de}}$ & $0.342^{\mathrm{h}}$ & $0.031^{\mathrm{fg}}$ & $0.110^{\mathrm{d}}$ & $1.47^{\text {ghi }}$ & $0.287^{\text {ghij }}$ & $0.780^{\mathrm{f}}$ & $0.541^{\mathrm{fg}}$ \\
\hline $45 \times \mathrm{T} 3 \times 25 \mathrm{C}$ & $1.95^{\mathrm{de}}$ & $6.11^{\mathrm{ijk}}$ & $0.312^{\mathrm{c}}$ & $58.2^{\text {fghijkl }}$ & $1.81^{\mathrm{f}}$ & $7.64^{\mathrm{g}}$ & $0.052^{\mathrm{de}}$ & $0.453^{\text {gh }}$ & $0.028^{g}$ & $0.173^{\mathrm{d}}$ & $2.51^{\mathrm{ef}}$ & $0.371^{\text {efgh }}$ & $0.919^{\text {ef }}$ & $0.632^{\mathrm{fg}}$ \\
\hline $45 \times \mathrm{T} 3 \times 40 \mathrm{C}$ & $2.29^{\mathrm{cd}}$ & $10.3^{\text {fghijk }}$ & $0.412^{\mathrm{c}}$ & $69.2^{\text {cdefghi }}$ & $3.07^{\mathrm{ab}}$ & $49.6^{\text {def }}$ & $0.060^{\text {cde }}$ & $0.578^{\text {efgh }}$ & $0.035^{\mathrm{fg}}$ & $0.193^{\mathrm{d}}$ & $3.03^{\mathrm{de}}$ & $0.575^{\mathrm{bcd}}$ & $0.990^{\mathrm{ef}}$ & $0.545^{\mathrm{gg}}$ \\
\hline $45 \times \mathrm{T} 6 \times 3 \mathrm{C}$ & $1.05^{\text {fghijk }}$ & $5.74^{\mathrm{ijk}}$ & $0.315^{\mathrm{c}}$ & $64.8^{\text {defghij }}$ & $1.81^{\mathrm{f}}$ & $4.23^{\mathrm{g}}$ & $0.053^{\mathrm{de}}$ & $0.369^{\mathrm{h}}$ & $0.135^{\mathrm{def}}$ & $0.214^{\mathrm{d}}$ & $1.46^{\text {ghi }}$ & $0.348^{\text {fghi }}$ & $1.05^{\mathrm{ef}}$ & $0.433^{\mathrm{fg}}$ \\
\hline $45 \times \mathrm{T} 6 \times 25 \mathrm{C}$ & $2.72^{\mathrm{bc}}$ & $14.1^{\text {defgh }}$ & $0.353^{\mathrm{c}}$ & $85.0^{\text {bcdef }}$ & $2.15^{\text {def }}$ & $11.4^{\mathrm{g}}$ & $0.050^{\mathrm{de}}$ & $0.546^{\text {fgh }}$ & $0.088^{\mathrm{efg}}$ & $0.185^{\mathrm{d}}$ & $3.62^{\text {cd }}$ & $0.486^{\text {cdef }}$ & $1.11^{\mathrm{ef}}$ & $0.543^{\mathrm{fg}}$ \\
\hline $45 \times \mathrm{T} 6 \times 40 \mathrm{C}$ & $2.91^{\mathrm{bc}}$ & $18.1^{\text {bcdef }}$ & $0.470^{\mathrm{c}}$ & $101^{\mathrm{b}}$ & $2.96^{\mathrm{abc}}$ & $80.2^{\mathrm{bcd}}$ & $0.060^{\text {cde }}$ & $0.740^{\text {cdefg }}$ & $0.091^{\mathrm{efg}}$ & $0.209^{\mathrm{d}}$ & $3.95^{\mathrm{bc}}$ & $0.270^{\text {ghij }}$ & $1.78^{\text {cdef }}$ & $1.71^{\text {cde }}$ \\
\hline $45 \times \mathrm{T} 12 \times 3 \mathrm{C}$ & $0.954^{\text {fghijk }}$ & $9.52^{\text {ghijk }}$ & $0.317^{\mathrm{c}}$ & $61.7^{\text {efghijk }}$ & $1.96^{\mathrm{ef}}$ & $5.79^{\mathrm{g}}$ & $0.054^{\mathrm{de}}$ & $0.392^{\text {gh }}$ & $0.160^{\text {de }}$ & $0.195^{\mathrm{d}}$ & $1.43^{\text {ghij }}$ & $0.028^{1}$ & $0.756^{\mathrm{f}}$ & $0.721^{\mathrm{fg}}$ \\
\hline $45 \times \mathrm{T} 12 \times 25 \mathrm{C}$ & $3.38^{\mathrm{ab}}$ & $19.4^{\text {bcde }}$ & $0.381^{c}$ & $89.9^{\text {bcde }}$ & $2.78^{\mathrm{abcd}}$ & $14.8^{\mathrm{fg}}$ & $0.045^{\mathrm{e}}$ & $0.618^{\text {defgh }}$ & $0.108^{\mathrm{efg}}$ & $0.227^{\mathrm{d}}$ & $4.60^{\mathrm{ab}}$ & $0.750^{\mathrm{a}}$ & $1.35^{\mathrm{def}}$ & $1.12^{\operatorname{defg}}$ \\
\hline $45 \times \mathrm{T} 12 \times 40 \mathrm{C}$ & $3.63^{\mathrm{a}}$ & $23.9^{\mathrm{bc}}$ & $0.543^{\mathrm{c}}$ & $136^{\mathrm{a}}$ & $3.33^{\mathrm{a}}$ & $113^{\mathrm{b}}$ & $0.061^{\text {cde }}$ & $0.927^{\text {bcde }}$ & $0.092^{\text {efg }}$ & $0.268^{\mathrm{d}}$ & $5.01^{\mathrm{a}}$ & $0.363^{\text {efgh }}$ & $1.99^{\text {cde }}$ & $1.77^{\mathrm{cd}}$ \\
\hline $80 \times \mathrm{T} 1 \times 3 \mathrm{C}$ & $0.503^{\mathrm{jk}}$ & $11.0^{\text {fghijk }}$ & $0.426^{\mathrm{c}}$ & $34.8^{\mathrm{jkl}}$ & $0.025^{\mathrm{g}}$ & $2.84^{\mathrm{g}}$ & $0.050^{\mathrm{de}}$ & $0.618^{\operatorname{defgh}}$ & $0.045^{\mathrm{fg}}$ & $0.626^{\text {abc }}$ & $0.540^{\mathrm{jk}}$ & $0.124^{\mathrm{jkl}}$ & $0.960^{\text {ef }}$ & $0.453^{\mathrm{fg}}$ \\
\hline $80 \times \mathrm{T} 1 \times 25 \mathrm{C}$ & $0.610^{\mathrm{ijk}}$ & $10.4^{\text {fghijk }}$ & $0.896^{\mathrm{c}}$ & $72.0^{\text {bcdefghi }}$ & $0.084^{\mathrm{g}}$ & $29.2^{\text {efg }}$ & $0.049^{\mathrm{de}}$ & $0.849^{\text {bcdef }}$ & $0.110^{\text {efg }}$ & $0.571^{\mathrm{bc}}$ & $0.708^{\text {hijk }}$ & $0.183^{\mathrm{ijkl}}$ & $1.98^{\text {cde }}$ & $0.915^{\text {defs }}$ \\
\hline $80 \times \mathrm{T} 1 \times 40 \mathrm{C}$ & $0.848^{\text {fghijk }}$ & $16.0^{\operatorname{defg}}$ & $1.23^{\mathrm{c}}$ & $85.1^{\text {bcdef }}$ & $0.098^{\mathrm{g}}$ & $95.1^{\mathrm{bc}}$ & $0.080^{\mathrm{cd}}$ & $0.883^{\text {bdef }}$ & $0.129^{\operatorname{defg}}$ & $0.582^{\mathrm{abc}}$ & $1.05^{\text {ghijk }}$ & $0.241^{\mathrm{hijk}}$ & $2.25^{\mathrm{cd}}$ & $1.28^{\mathrm{def}}$ \\
\hline $80 \times \mathrm{T} 3 \times 3 \mathrm{C}$ & $0.369^{\mathrm{k}}$ & $7.87^{\mathrm{hijk}}$ & $0.428^{\mathrm{c}}$ & $29.8^{1}$ & $0.029^{g}$ & $9.64^{\mathrm{g}}$ & $0.041^{\mathrm{e}}$ & $0.552^{\mathrm{fgh}}$ & $0.041^{\mathrm{fg}}$ & $0.458^{\mathrm{c}}$ & $0.487^{\mathrm{k}}$ & $0.147^{\mathrm{jkl}}$ & $1.03^{\mathrm{ef}}$ & $0.450^{\mathrm{fg}}$ \\
\hline $80 \times \mathrm{T} 3 \times 25 \mathrm{C}$ & $0.772^{\text {ghijk }}$ & $16.1^{\text {cdefg }}$ & $1.03^{\mathrm{c}}$ & $82.3^{\text {bcdefg }}$ & $0.067^{g}$ & $59.1^{\mathrm{de}}$ & $0.051^{\mathrm{de}}$ & $1.02^{\mathrm{bc}}$ & $0.218^{\mathrm{cd}}$ & $0.629^{\mathrm{abc}}$ & $0.790^{\mathrm{hijk}}$ & $0.189^{\mathrm{ijkl}}$ & $2.25^{\mathrm{cd}}$ & $1.14^{\text {defg }}$ \\
\hline $80 \times \mathrm{T} 3 \times 40 \mathrm{C}$ & $1.00^{\text {fghijk }}$ & $25.4^{\mathrm{b}}$ & $3.88^{\mathrm{b}}$ & $94.3^{\text {bcd }}$ & $0.114^{\mathrm{g}}$ & $168^{\mathrm{a}}$ & $0.091^{\mathrm{c}}$ & $1.47^{\mathrm{a}}$ & $0.443^{\mathrm{b}}$ & $0.640^{\mathrm{ab}}$ & $1.26^{\text {ghijk }}$ & $0.470^{\text {cdef }}$ & $2.83^{\mathrm{c}}$ & $3.98^{\mathrm{b}}$ \\
\hline $80 \times \mathrm{T} 6 \times 3 \mathrm{C}$ & $0.554^{\mathrm{jk}}$ & $12.2^{\text {efghij }}$ & $0.565^{\mathrm{c}}$ & $44.3^{\mathrm{ijk} k}$ & $0.037^{\mathrm{g}}$ & $10.3^{g}$ & $0.055^{\mathrm{de}}$ & $0.733^{\text {cdefg }}$ & $0.056^{\mathrm{efg}}$ & $0.600^{\mathrm{abc}}$ & $0.613^{\mathrm{ijk}}$ & $0.239^{\mathrm{hijk}}$ & $1.39^{\operatorname{def}}$ & $0.763^{\text {efg }}$ \\
\hline $80 \times \mathrm{T} 6 \times 25 \mathrm{C}$ & $0.931^{\text {fghijk }}$ & $20.7^{\mathrm{bcd}}$ & $1.11^{\mathrm{c}}$ & $89.1^{\text {bcde }}$ & $0.064^{\mathrm{g}}$ & $80.6^{\text {bcd }}$ & $0.060^{\text {cde }}$ & $1.11^{\mathrm{b}}$ & $0.273^{\mathrm{c}}$ & $0.697^{\mathrm{ab}}$ & $0.878^{\text {ghijk }}$ & $0.240^{\mathrm{hijk}}$ & $2.37^{\mathrm{cd}}$ & $1.22^{\text {defg }}$ \\
\hline $80 \times \mathrm{T} 6 \times 40 \mathrm{C}$ & $1.15^{\text {fghij }}$ & $43.4^{\mathrm{a}}$ & $3.70^{\mathrm{b}}$ & $98.6^{\mathrm{bc}}$ & $0.099^{g}$ & $191^{\mathrm{a}}$ & $0.158^{\mathrm{b}}$ & $1.72^{\mathrm{a}}$ & $0.629^{\mathrm{a}}$ & $0.737^{\mathrm{ab}}$ & $1.31^{\text {ghijk }}$ & $0.611^{\mathrm{abc}}$ & $5.60^{\mathrm{b}}$ & $4.22^{\mathrm{b}}$ \\
\hline $80 \times \mathrm{T} 12 \times 3 \mathrm{C}$ & $0.663^{\mathrm{hijk}}$ & $13.2^{\text {defghi }}$ & $6.68^{\mathrm{a}}$ & $52.4^{\text {ghijkl }}$ & $0.046^{\mathrm{g}}$ & $64.3^{\text {cd }}$ & $0.061^{\text {cde }}$ & $0.857^{\text {bcdef }}$ & $0.073^{\text {efg }}$ & $0.649^{\mathrm{ab}}$ & $0.707^{\mathrm{hijk}}$ & $0.341^{\text {fghi }}$ & $1.62^{\text {def }}$ & $2.58^{\mathrm{c}}$ \\
\hline $80 \times \mathrm{T} 12 \times 25 \mathrm{C}$ & $0.824^{\text {fghijk }}$ & $17.7^{\text {bcdef }}$ & $0.928^{\mathrm{c}}$ & $75.0^{\text {bcdefgh }}$ & $0.085^{g}$ & $71.0^{\mathrm{cd}}$ & $0.052^{\mathrm{de}}$ & $0.952^{\mathrm{bcd}}$ & $0.297^{\mathrm{c}}$ & $0.587^{\mathrm{abc}}$ & $0.724^{\mathrm{hijk}}$ & $0.187^{\mathrm{ijkl}}$ & $1.97^{\text {cde }}$ & $2.45^{\mathrm{c}}$ \\
\hline $80 \times \mathrm{T} 12 \times 40 \mathrm{C}$ & $1.50^{\mathrm{ef}}$ & $44.5^{\mathrm{a}}$ & $6.25^{\mathrm{a}}$ & $99.9^{\mathrm{b}}$ & $0.088^{g}$ & $197^{\mathrm{a}}$ & $0.223^{\mathrm{a}}$ & $1.81^{\mathrm{a}}$ & $0.720^{\mathrm{a}}$ & $0.750^{\mathrm{a}}$ & $1.35^{\text {ghijk }}$ & $0.685^{\mathrm{ab}}$ & $8.17^{\mathrm{a}}$ & $7.86^{\mathrm{a}}$ \\
\hline Time $\times$ temper & ture & & & & & & & & & & & & & \\
\hline $\mathrm{T} 1 \times 3 \mathrm{C}$ & $0.886^{\mathrm{f}}$ & $7.24^{\mathrm{cd}}$ & $0.369^{\mathrm{c}}$ & $33.3^{\mathrm{f}}$ & $1.16^{\text {bcde }}$ & $2.18^{\mathrm{f}}$ & $0.051^{\mathrm{d}}$ & $0.471^{\mathrm{f}}$ & $0.065^{\mathrm{de}}$ & $0.425^{\mathrm{ab}}$ & $1.03^{\mathrm{e}}$ & $0.278^{\text {cde }}$ & $1.03^{\mathrm{ef}}$ & $0.361^{\mathrm{d}}$ \\
\hline $\mathrm{T} 1 \times 25 \mathrm{C}$ & $0.969^{\text {ef }}$ & $7.07^{\mathrm{cd}}$ & $0.596^{\mathrm{c}}$ & $53.6^{\mathrm{de}}$ & $1.25^{\text {abcde }}$ & $16.7^{\mathrm{ef}}$ & $0.054^{\mathrm{d}}$ & $0.622^{\text {def }}$ & $0.091^{\mathrm{de}}$ & $0.403^{\mathrm{ab}}$ & $1.13^{\mathrm{de}}$ & $0.353^{\text {bc }}$ & $1.67^{\text {cde }}$ & $0.735^{\mathrm{d}}$ \\
\hline $\mathrm{T} 1 \times 40 \mathrm{C}$ & $1.11^{\mathrm{ef}}$ & $10.6^{\mathrm{cd}}$ & $0.763^{\mathrm{c}}$ & $58.0^{\mathrm{d}}$ & $1.37^{\text {abcde }}$ & $55.5^{\mathrm{c}}$ & $0.065^{\mathrm{cd}}$ & $0.614^{\text {def }}$ & $0.104^{\mathrm{d}}$ & $0.389^{\mathrm{bc}}$ & $1.40^{\mathrm{de}}$ & $0.164^{\mathrm{f}}$ & $1.77^{\mathrm{cd}}$ & $0.920^{\mathrm{d}}$ \\
\hline $\mathrm{T} 3 \times 3 \mathrm{C}$ & $0.755^{\mathrm{f}}$ & $6.22^{\mathrm{d}}$ & $0.369^{\mathrm{c}}$ & $38.9^{\text {ef }}$ & $0.930^{\mathrm{e}}$ & $6.40^{\mathrm{f}}$ & $0.047^{\mathrm{d}}$ & $0.447^{\mathrm{f}}$ & $0.036^{\mathrm{e}}$ & $0.284^{\mathrm{c}}$ & $0.982^{\mathrm{e}}$ & $0.217^{\mathrm{def}}$ & $0.907^{\mathrm{f}}$ & $0.495^{\mathrm{d}}$ \\
\hline $\mathrm{T} 3 \times 25 \mathrm{C}$ & $1.36^{\mathrm{de}}$ & $11.1^{\text {cd }}$ & $0.674^{\mathrm{c}}$ & $70.2^{\mathrm{cd}}$ & $0.940^{\mathrm{e}}$ & $33.3^{\mathrm{de}}$ & $0.051^{\mathrm{d}}$ & $0.741^{\mathrm{de}}$ & $0.123^{\mathrm{cd}}$ & $0.401^{\mathrm{ab}}$ & $1.65^{\text {cd }}$ & $0.280^{\text {cde }}$ & $1.58^{\text {cdef }}$ & $0.886^{\mathrm{d}}$ \\
\hline $\mathrm{T} 3 \times 40 \mathrm{C}$ & $1.64^{\mathrm{cd}}$ & $17.8^{\mathrm{b}}$ & $2.14^{\mathrm{b}}$ & $81.7^{\mathrm{bc}}$ & $1.59^{\mathrm{ab}}$ & $109^{b}$ & $0.075^{\mathrm{c}}$ & $1.02^{\mathrm{bc}}$ & $0.239^{\mathrm{b}}$ & $0.417^{\mathrm{ab}}$ & $2.14^{\mathrm{bc}}$ & $0.523^{\mathrm{a}}$ & $1.91^{\mathrm{c}}$ & $2.265^{\mathrm{c}}$ \\
\hline $\mathrm{T} 6 \times 3 \mathrm{C}$ & $0.803^{\mathrm{f}}$ & $8.98^{\mathrm{cd}}$ & $0.440^{\mathrm{c}}$ & $54.6^{\mathrm{de}}$ & $0.925^{\mathrm{e}}$ & $7.27^{\mathrm{f}}$ & $0.054^{\mathrm{d}}$ & $0.551^{\mathrm{ef}}$ & $0.095^{\mathrm{de}}$ & $0.407^{\mathrm{ab}}$ & $1.03^{\mathrm{e}}$ & $0.294^{\mathrm{cd}}$ & $1.22^{\text {cdef }}$ & $0.598^{\mathrm{d}}$ \\
\hline $\mathrm{T} 6 \times 25 \mathrm{C}$ & $1.82^{\mathrm{bc}}$ & $17.4^{\mathrm{b}}$ & $0.731^{\mathrm{c}}$ & $87.0^{\mathrm{bc}}$ & $1.10^{\text {cde }}$ & $46.0^{\text {cd }}$ & $0.055^{\mathrm{cd}}$ & $0.829^{\mathrm{cd}}$ & $0.181^{\mathrm{bc}}$ & $0.441^{\mathrm{ab}}$ & $2.25^{\mathrm{b}}$ & $0.363^{\mathrm{bc}}$ & $1.74^{\mathrm{cd}}$ & $0.881^{\mathrm{d}}$ \\
\hline $\mathrm{T} 6 \times 40 \mathrm{C}$ & $2.03^{\mathrm{bc}}$ & $30.7^{\mathrm{a}}$ & $2.08^{\mathrm{b}}$ & $99.8^{\mathrm{ab}}$ & $1.53^{\mathrm{abc}}$ & $135^{\mathrm{a}}$ & $0.109^{b}$ & $1.23^{\mathrm{ab}}$ & $0.360^{\mathrm{a}}$ & $0.473^{\mathrm{ab}}$ & $2.63^{\mathrm{ab}}$ & $0.440^{\mathrm{ab}}$ & $3.69^{\mathrm{b}}$ & $2.97^{\mathrm{b}}$ \\
\hline $\mathrm{T} 12 \times 3 \mathrm{C}$ & $0.808^{\mathrm{f}}$ & $11.3^{\mathrm{c}}$ & $3.49^{\mathrm{a}}$ & $57.1^{\mathrm{de}}$ & $1.00^{\mathrm{de}}$ & $35.0^{\text {cde }}$ & $0.058^{\text {cd }}$ & $0.625^{\text {def }}$ & $0.117^{\mathrm{cd}}$ & $0.422^{\mathrm{ab}}$ & $1.07^{\mathrm{de}}$ & $0.184^{\mathrm{ef}}$ & $1.18^{\mathrm{def}}$ & $1.65^{\mathrm{c}}$ \\
\hline $\mathrm{T} 12 \times 25 \mathrm{C}$ & $2.10^{\mathrm{b}}$ & $18.5^{\mathrm{b}}$ & $0.655^{\mathrm{c}}$ & $82.5^{\mathrm{bc}}$ & $1.43^{\mathrm{abcd}}$ & $42.9^{\mathrm{cd}}$ & $0.049^{\mathrm{d}}$ & $0.785^{\mathrm{d}}$ & $0.203^{\mathrm{b}}$ & $0.407^{\mathrm{ab}}$ & $2.66^{\mathrm{ab}}$ & $0.468^{\mathrm{a}}$ & $1.66^{\text {cde }}$ & $1.79^{\mathrm{c}}$ \\
\hline $\mathrm{T} 12 \times 40 \mathrm{C}$ & $2.56^{\mathrm{a}}$ & $34.2^{\mathrm{a}}$ & $3.39^{\mathrm{a}}$ & $118^{\mathrm{a}}$ & $1.71^{\mathrm{a}}$ & $155^{\mathrm{a}}$ & $0.142^{\mathrm{a}}$ & $1.37^{\mathrm{a}}$ & $0.406^{\mathrm{a}}$ & $0.509^{\mathrm{a}}$ & $3.18^{\mathrm{a}}$ & $0.524^{\mathrm{a}}$ & $5.08^{\mathrm{a}}$ & $4.81^{\mathrm{a}}$ \\
\hline Protein $\times$ temp & rature & & & & & & & & & & & & & \\
\hline $45 \times 3 \mathrm{C}$ & $1.10^{\mathrm{b}}$ & $5.82^{\mathrm{d}}$ & $0.313^{\mathrm{d}}$ & $51.6^{\mathrm{d}}$ & $1.97^{\mathrm{c}}$ & $3.67^{\mathrm{d}}$ & $0.053^{\mathrm{b}}$ & $0.357^{\mathrm{e}}$ & $0.103^{c}$ & $0.186^{\mathrm{c}}$ & $1.47^{\mathrm{c}}$ & $0.274^{\mathrm{bc}}$ & $0.927^{\mathrm{d}}$ & $0.491^{\mathrm{d}}$ \\
\hline $45 \times 25 \mathrm{C}$ & $2.34^{\mathrm{a}}$ & $10.8^{\mathrm{c}}$ & $0.336^{\mathrm{d}}$ & $67.1^{\mathrm{c}}$ & $2.29^{\mathrm{b}}$ & $9.58^{\mathrm{cd}}$ & $0.051^{\mathrm{b}}$ & $0.503^{\mathrm{d}}$ & $0.074^{\mathrm{cd}}$ & $0.205^{\mathrm{c}}$ & $3.07^{\mathrm{b}}$ & $0.532^{\mathrm{a}}$ & $1.18^{\mathrm{cd}}$ & $0.714^{\mathrm{cd}}$ \\
\hline $45 \times 40 \mathrm{C}$ & $2.55^{\mathrm{a}}$ & $14.4^{\mathrm{b}}$ & $0.430^{\mathrm{d}}$ & $84.5^{\mathrm{ab}}$ & $3.00^{\mathrm{a}}$ & $64.9^{\mathrm{b}}$ & $0.057^{\mathrm{b}}$ & $0.648^{\mathrm{c}}$ & $0.074^{\mathrm{cd}}$ & $0.217^{\mathrm{c}}$ & $3.44^{\mathrm{a}}$ & $0.324^{\mathrm{b}}$ & $1.51^{\mathrm{c}}$ & $1.14^{\mathrm{b}}$ \\
\hline $80 \times 3 \mathrm{C}$ & $0.522^{\mathrm{c}}$ & $11.0^{\mathrm{c}}$ & $2.025^{\mathrm{b}}$ & $40.3^{\mathrm{d}}$ & $0.034^{\mathrm{d}}$ & $21.7^{\mathrm{c}}$ & $0.052^{\mathrm{b}}$ & $0.690^{\mathrm{c}}$ & $0.054^{\mathrm{d}}$ & $0.583^{\mathrm{b}}$ & $0.587^{\mathrm{d}}$ & $0.213^{\text {cd }}$ & $1.25^{\mathrm{cd}}$ & $1.06^{\mathrm{bc}}$ \\
\hline $80 \times 25 \mathrm{C}$ & $0.784^{\mathrm{c}}$ & $16.2^{\mathrm{b}}$ & $0.992^{c}$ & $79.6^{\mathrm{b}}$ & $0.075^{\mathrm{d}}$ & $60.0^{\mathrm{b}}$ & $0.053^{\mathrm{b}}$ & $0.985^{\mathrm{b}}$ & $0.225^{\mathrm{b}}$ & $0.621^{\mathrm{ab}}$ & $0.775^{\mathrm{d}}$ & $0.200^{\mathrm{d}}$ & $2.14^{\mathrm{b}}$ & $1.43^{\mathrm{b}}$ \\
\hline $80 \times 40 \mathrm{C}$ & $1.12^{\mathrm{b}}$ & $32.3^{\mathrm{a}}$ & $3.768^{\mathrm{a}}$ & $94.5^{\mathrm{a}}$ & $0.100^{\mathrm{d}}$ & $163^{\mathrm{a}}$ & $0.138^{\mathrm{a}}$ & $1.47^{\mathrm{a}}$ & $0.480^{\mathrm{a}}$ & $0.677^{\mathrm{a}}$ & $1.24^{\mathrm{c}}$ & $0.502^{\mathrm{a}}$ & $4.71^{\mathrm{a}}$ & $4.33^{\mathrm{a}}$ \\
\hline ein $\times$ time & & & & & & & & & & & & & & \\
\hline $45 \times \mathrm{T} 1$ & $1.32^{\mathrm{d}}$ & $4.15^{\mathrm{d}}$ & $0.301^{\mathrm{d}}$ & $32.7^{\mathrm{e}}$ & $2.45^{\mathrm{ab}}$ & $7.24^{\mathrm{e}}$ & $0.053^{\mathrm{c}}$ & $0.355^{\mathrm{f}}$ & $0.079^{\mathrm{cd}}$ & $0.219^{\mathrm{d}}$ & $1.61^{\mathrm{d}}$ & $0.348^{\mathrm{ab}}$ & $1.25^{\mathrm{de}}$ & $0.459^{\mathrm{d}}$ \\
\hline $45 \times \mathrm{T} 3$ & $1.79^{\mathrm{c}}$ & $7.01^{\mathrm{d}}$ & $0.344^{\mathrm{d}}$ & $58.5^{\mathrm{d}}$ & $2.24^{\mathrm{b}}$ & $20.1^{\mathrm{de}}$ & $0.054^{\mathrm{c}}$ & $0.458^{\text {ef }}$ & $0.032^{\mathrm{d}}$ & $0.159^{\mathrm{d}}$ & $2.34^{\mathrm{c}}$ & $0.411^{\mathrm{a}}$ & $0.897^{\mathrm{e}}$ & $0.572^{\mathrm{d}}$ \\
\hline $45 \times \mathrm{T} 6$ & $2.22^{\mathrm{b}}$ & $12.6^{\mathrm{c}}$ & $0.379^{\text {cd }}$ & $83.6^{\mathrm{ab}}$ & $2.31^{\mathrm{b}}$ & $31.9^{\text {cd }}$ & $0.054^{\mathrm{c}}$ & $0.552^{\mathrm{de}}$ & $0.105^{\mathrm{c}}$ & $0.203^{\mathrm{d}}$ & $3.01^{\mathrm{b}}$ & $0.368^{\mathrm{a}}$ & $1.31^{\mathrm{de}}$ & $0.898^{\mathrm{cd}}$ \\
\hline$\times \mathrm{T} 12$ & $2.65^{\mathrm{a}}$ & $17.6^{\mathrm{b}}$ & $0.414^{\text {cd }}$ & $96.1^{\mathrm{a}}$ & $2.69^{\mathrm{a}}$ & $44.8^{\mathrm{c}}$ & $0.053^{\mathrm{c}}$ & $0.646^{\mathrm{cd}}$ & $0.120^{c}$ & $0.230^{\mathrm{d}}$ & $3.68^{\mathrm{a}}$ & $0.380^{\mathrm{a}}$ & $1.36^{\mathrm{de}}$ & $1.20^{\mathrm{c}}$ \\
\hline$\times \mathrm{T} 1$ & $0.654^{\mathrm{f}}$ & $12.4^{\mathrm{c}}$ & $0.851^{\mathrm{c}}$ & $64.0^{\text {cd }}$ & $0.069^{\mathrm{c}}$ & $42.4^{\mathrm{c}}$ & $0.060^{c}$ & $0.783^{\mathrm{c}}$ & $0.094^{\mathrm{c}}$ & $0.593^{\mathrm{bc}}$ & $0.767^{\mathrm{e}}$ & $0.183^{\mathrm{c}}$ & $1.73^{\mathrm{cd}}$ & $0.884^{\mathrm{cd}}$ \\
\hline $0 \times \mathrm{T} 3$ & $0.713^{\mathrm{ef}}$ & $16.4^{\mathrm{b}}$ & $1.78^{\mathrm{b}}$ & $68.8^{\mathrm{cd}}$ & $0.070^{\mathrm{c}}$ & $79.1^{\mathrm{b}}$ & $0.061^{c}$ & $1.01^{\mathrm{b}}$ & $0.234^{\mathrm{b}}$ & $0.575^{\mathrm{c}}$ & $0.848^{\mathrm{e}}$ & $0.269^{\mathrm{b}}$ & $2.04^{\mathrm{c}}$ & $1.85^{\mathrm{b}}$ \\
\hline
\end{tabular}


and 80 stored at $40^{\circ} \mathrm{C}$ became noticeably browner at 3 mo, whereas it was 6 mo before both MPC 45 and 80 stored at $25^{\circ} \mathrm{C}$ became noticeably brown. This visual color change corresponded to an increase in furosine content of approximately 400 to $500 \%$ (Figure 2).

Solubility of MPC During Storage. Temperature, storage time, and protein level had an effect on solubility of MPC powders $(P<0.05)$ (Figure 3$)$. The solubility of MPC 45 stored at 3 and $25^{\circ} \mathrm{C}$ was reduced by 9 and $15 \%$, respectively, over 12 mo whereas the solubility of MPC 45 stored at $40^{\circ} \mathrm{C}$ decreased by approximately $28 \%$ (Figure 3 ). Temperature had a more pronounced effect on the solubility of MPC 80 than time. The solubility of MPC 80 stored at $3^{\circ} \mathrm{C}$ was reduced by approximately $15 \%$ after 12 mo, whereas the solubility of MPC 80 stored at $25^{\circ} \mathrm{C}$ was reduced by $51 \%$ and at $40^{\circ} \mathrm{C}$, solubility was reduced by $88 \%$.

The solubility of MPC has been extensively studied (Schuck et al., 1994; Anema et al., 2006; Havea 2006; Baldwin and Truong, 2007; Le et al., 2011a,b) Interestingly, whereas past studies agree that solubility of stored MPC is an issue, the solubility of MPC during storage is highly variable and dependent on many factors. For instance, Havea (2006) produced 6 MPC 85 powders, one of which had a solubility of $53 \%$ after 2 $\mathrm{d}$, whereas another, which had had $30 \%$ of its calcium replaced by sodium ions, had a solubility of $98 \%$ after 7 wk of storage at $20^{\circ} \mathrm{C}$. Anema et al. (2006) tested the solubility of MPC 85 at temperatures ranging from $20^{\circ}$ to $50^{\circ} \mathrm{C}$ of which, after $60 \mathrm{~d}$ of storage, the $20^{\circ} \mathrm{C}$ treatment retained $100 \%$ solubility, whereas the $50^{\circ} \mathrm{C}$ treatment dropped to approximately $20 \%$ solubility after 10 d. Le et al. (2011a) studied the effect of relative humidity (RH) and temperature on MPC 80 solubility and demonstrated a $50 \%$ drop in solubility of MPC 85 stored at $25^{\circ} \mathrm{C}$ with a $\mathrm{RH}$ of $44 \%$ (the $\mathrm{RH}$ used in this study) after 12 wk. The solubility of MPC 80 stored at $40^{\circ} \mathrm{C}$ with a $\mathrm{RH}$ of $44 \%$ decreased to approximately $20 \%$ after $4 \mathrm{wk}$. Whereas the variables in these previous studies vary, the current study solubility results are consistent with past studies, especially Le et al. (2011a), whose storage conditions matched those of the current study (except for the lack of a $3^{\circ} \mathrm{C}$ storage treatment). Ultimately, the purpose of experiment 2 was to determine the shelf life of MPC 80 stored under specific conditions, and although flavor is of utmost importance to the shelf life of any food product, usability, specifically solubility in the case of MPC 80 must also be taken into account. These results confirm that temperature abuse during storage for a short period renders MPC virtually unusable in liquid suspensions. Solubility and furosine content were inversely correlated $\left(\mathrm{R}^{2}=0.84-0.97, P<\right.$ $0.05)$, which suggests that Maillard browning products 
Table 7. Sensory profiles of milk protein concentrates (MPC45 and 80) stored for $0,1,3,6$, and 12 mo at 3,25 , and $40^{\circ} \mathrm{C}^{1}$

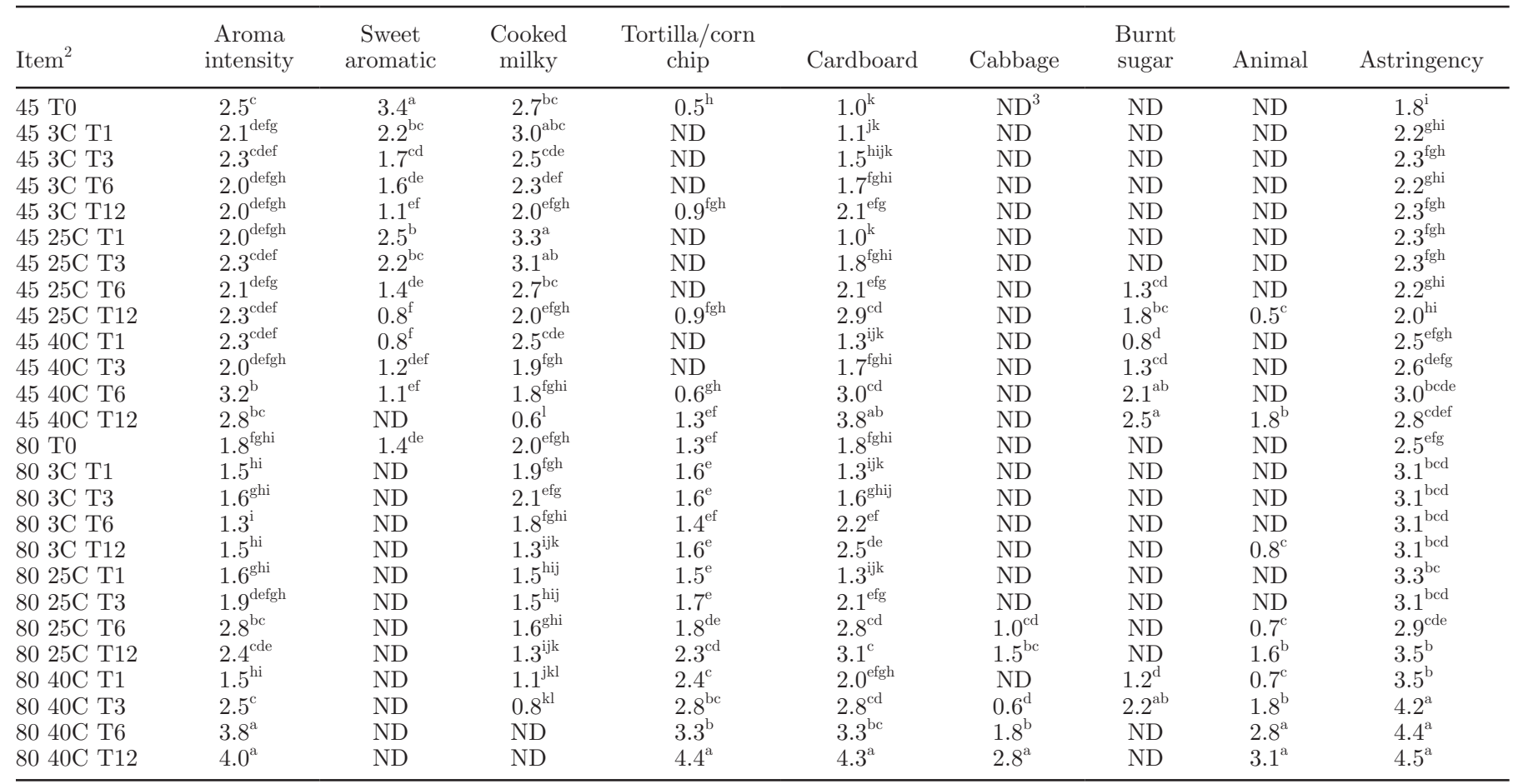

${ }^{\mathrm{a}-\mathrm{l}}$ Means in a column not sharing a common superscript are different $(P<0.05)$.

${ }^{1}$ Attribute intensities were scored on a 0- to 15-point universal intensity scale (Meilgaard et al., 2009). Most dried ingredient flavors fall between 0 and 4 (Croissant et al., 2009; Wright et al., 2009).

${ }^{2} \mathrm{~T} 1=1$ mo storage, $\mathrm{T} 3=3 \mathrm{mo}$ storage, $\mathrm{T} 6=6$ mo storage, $\mathrm{T} 12=12$ mo storage. $3 \mathrm{C}=$ stored at $3{ }^{\circ} \mathrm{C}, 25 \mathrm{C}=$ stored at $25^{\circ} \mathrm{C}, 40 \mathrm{C}=$ stored at $40^{\circ} \mathrm{C}$.

${ }^{3} \mathrm{ND}=$ not detected.

are reacting with AA, causing cross-linking of proteins and subsequent solubility loss.

Instrumental Volatile and Descriptive Analysis of MPC During Storage. Storage time, temperature, and protein content also affected volatile compounds and flavor of MPC powders. Aldehydes (hexanal, heptanal, octanal, nonanal, methional, 2 and 3-methylbutanal, E,E 2,4 decadienal) and other lipid oxidation products such as 2-pentylfuran and 1-octen-3-one and sulfur-containing volatile compounds (dimethyl sulfide, dimethyl disulfide, dimethyl trisulfide) increased with time and as storage temperature increased in both MPC 45 and MPC 80 (Table 6).

Generally, MPC 45 stored at lower temperatures for less time were associated with sweet aromatic and cooked milky flavors and a sweet taste. As the powders aged, aroma intensity, cardboard, animal, and tortilla/ corn chip flavors increased. The MPC 45 stored at $25^{\circ} \mathrm{C}$ exhibited a burnt sugar flavor after 6 mo, whereas MPC 45 stored at $40^{\circ} \mathrm{C}$ exhibited this after only $1 \mathrm{mo}$ of storage. No burnt sugar flavor was detected in MPC 45 stored at $3^{\circ} \mathrm{C}$. The MPC 45 stored at 25 and $40^{\circ} \mathrm{C}$ exhibited an animal flavor after 12 mo, whereas this flavor was not detected in product stored at $3{ }^{\circ} \mathrm{C}$ (Table 7). Because burnt sugar flavor followed the trend of Maillard reactions (furosine), it seems likely that this flavor can be attributed to Maillard reaction products such as sotolon or furaneol. These compounds are formed by thermal treatment of sugars and have distinct burnt sugary aromas, and have been previously reported in stored SMP (Karagul-Yuceer et al., 2002), sweet whey powder (Mahajan et al., 2004), and whey protein (Carunchia Whetstine et al., 2005) extracted by solvent extraction. These compounds were also odor active in fresh MPC 80 in experiment 1 by solvent extraction, but not by SPME, so they likely were not well extracted by SPME. Additional work would be needed to confirm this hypothesis. Cardboard flavor in whey protein concentrates has been sourced to pentanal, heptanal, and nonanal in the presence of DMTS and 1-octen-3-one, and lipid oxidation compound increases are the likely source of cardboard flavor in the stored milk proteins (Whitson et al., 2010).

Similar to MPC 45, MPC 80 stored at lower temperatures for less time were associated with sweet aromatic and cooked milky flavors. As the powders aged, overall 
aroma intensity, cardboard, animal, cabbage, and tortilla/corn chip flavors increased. This result is consistent with an increase in the lipid oxidation and protein degradation volatile compounds that cause these offflavors. Cabbage flavor in whey protein isolate has been sourced to DMTS (Wright et al., 2006). Compounds attributed to cardboard and cabbage flavor increased during storage (pentanal, heptanal, nonanal, 1-octen3-one, and DMTS, Table 6). The compounds, E,E 2,4 decadienal, 3-methylbutanal, methional, and hexanal, possible sources of corn chip, fatty, and potato flavors, increased during storage in MPC 80 and 45. The MPC 80 stored at $3^{\circ} \mathrm{C}$ exhibited an animal flavor after 12 mo, whereas MPC 80 stored at 25 and $40^{\circ} \mathrm{C}$ exhibited this flavor after 6 and 1 mo, respectively. The MPC 80 stored at $25^{\circ} \mathrm{C}$ exhibited a cabbage flavor after $6 \mathrm{mo}$, but burnt sugar flavor was not detected. The MPC 80 stored at $40^{\circ} \mathrm{C}$ exhibited cabbage flavor after 3 mo and burnt sugar flavor after 1 mo. These 2 flavors were not detected in MPC 80 stored at $3^{\circ} \mathrm{C}$. Unlike the MPC

\section{Biplot (axes F1 and F2: 75 \%)}

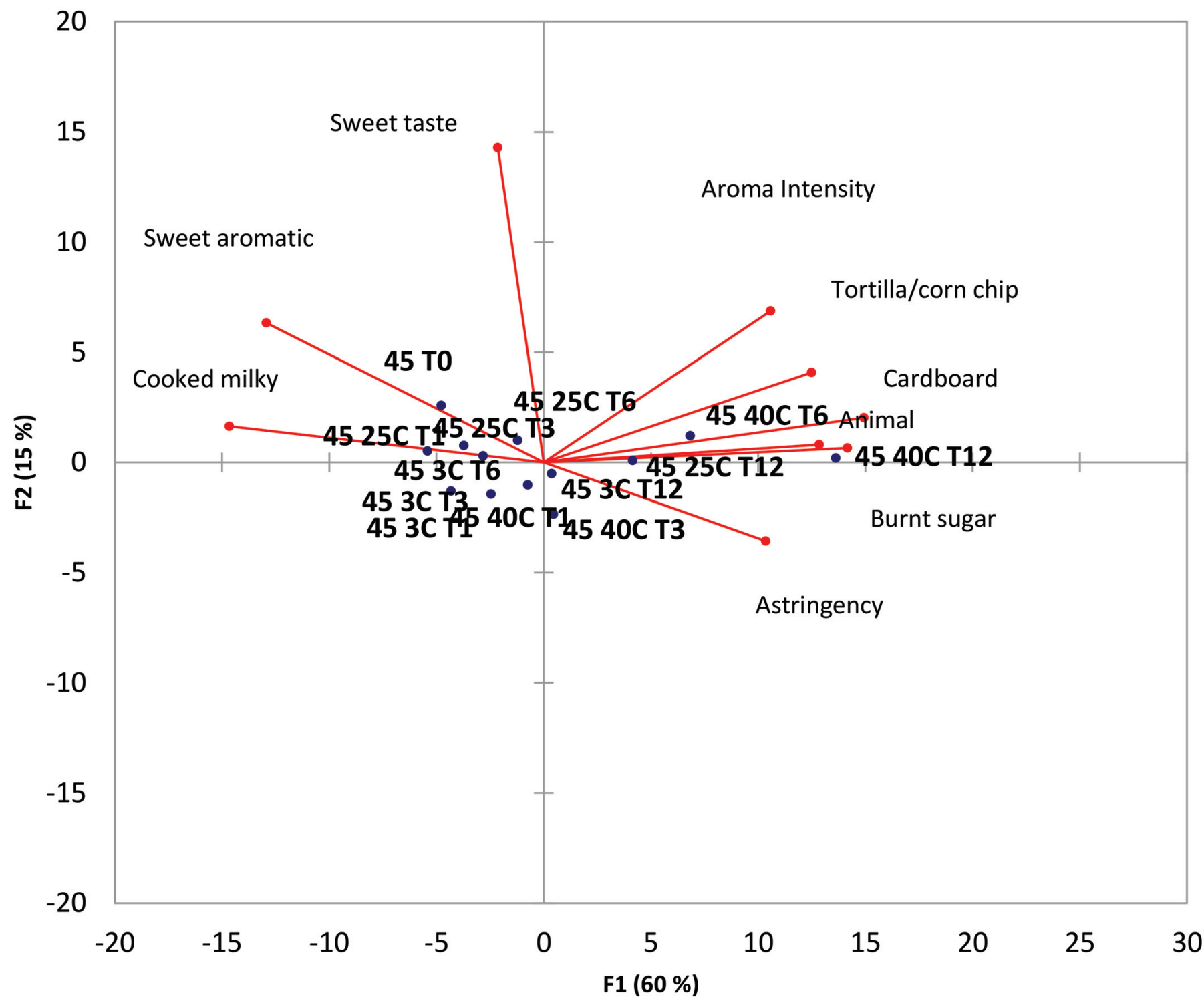

Figure 4. Principal component biplot of sensory attributes of milk protein concentrate (MPC) 45 stored at 3,25 , and $40^{\circ} \mathrm{C}$ for $0,1,3,6$, and 12 mo. $\mathrm{T} 0=$ no storage, $\mathrm{T} 1=1 \mathrm{mo}$ storage, $\mathrm{T} 3=3$ mo storage, $\mathrm{T} 6=6 \mathrm{mo}$ storage, $\mathrm{T} 12=12$ mo storage. $3 \mathrm{C}=$ stored at $3{ }^{\circ} \mathrm{C}, 25 \mathrm{C}=$ stored at $25^{\circ} \mathrm{C}, 40 \mathrm{C}=$ stored at $40^{\circ} \mathrm{C}$. Color version available online. 
Biplot (axes F1 and F2: 85 \%)

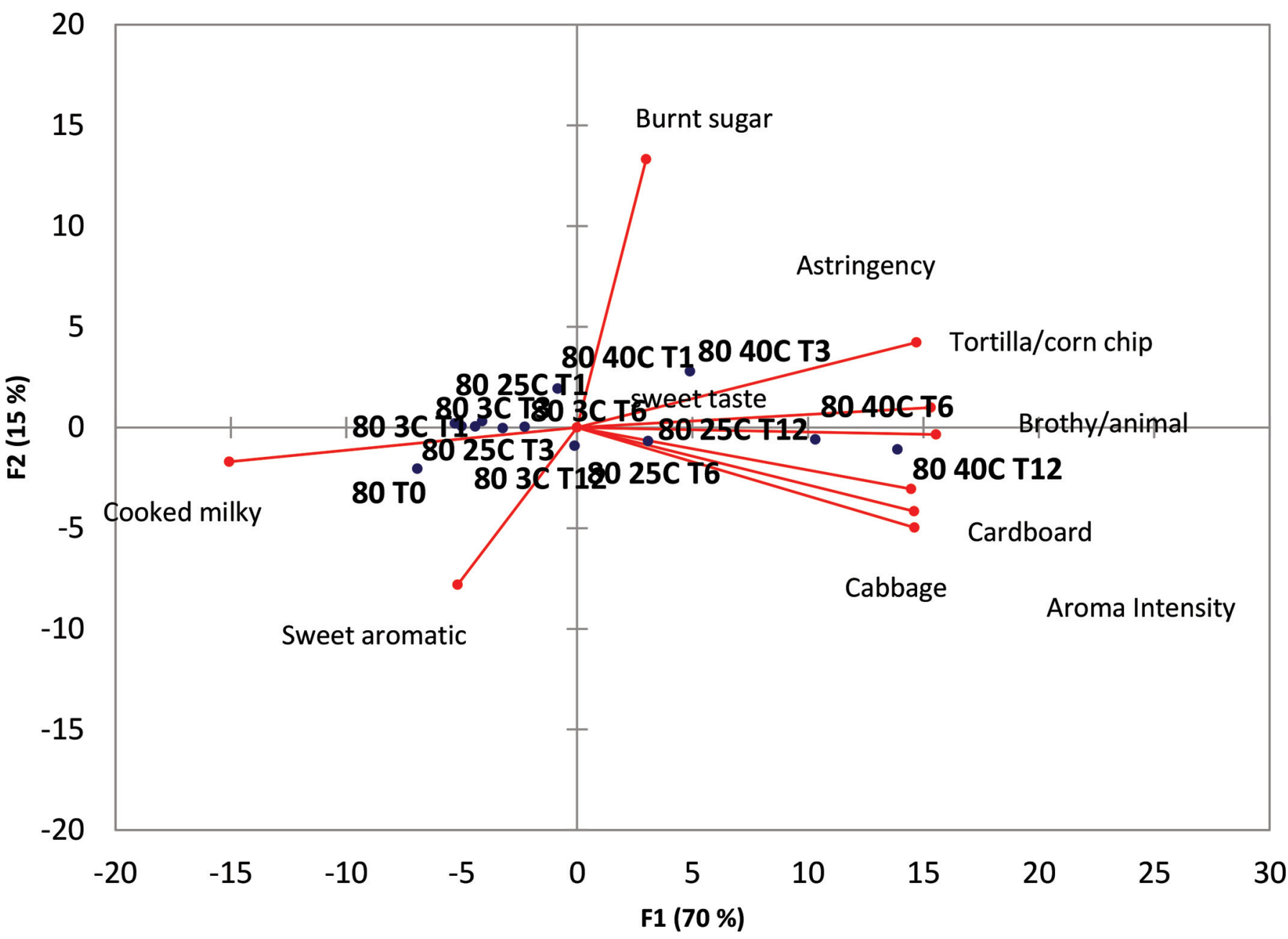

Figure 5. Principal component biplot of sensory attributes of milk protein concentrate (MPC) 80 stored at 3,25 , and $40^{\circ} \mathrm{C}$ for $0,1,3,6$, and 12 mo. T0 $=$ no storage, $\mathrm{T} 1=1 \mathrm{mo}$ storage, $\mathrm{T} 3=3$ mo storage, $\mathrm{T} 6=6$ mo storage, $\mathrm{T} 12=12$ mo storage. $3 \mathrm{C}=$ stored at $3{ }^{\circ} \mathrm{C}, 25 \mathrm{C}=$ stored at $25^{\circ} \mathrm{C}, 40 \mathrm{C}=$ stored at $40^{\circ} \mathrm{C}$. Color version available online.

45, MPC 80 stored at all 3 temperatures exhibited the tortilla/corn chip flavor immediately after production rather than following storage.

The principal component biplots generated from sensory analysis and relative abundance means can be used to approximate similarities in the stage of shelf-life of the MPC 45 and 80 powders by the increase in animal, tortilla/corn chip, cardboard, cabbage, and aroma intensity and increase in corresponding lipid oxidation and heat-induced volatile compound concentration over time (Figures 4, 5, 6, and 7). For instance, MPC 45 stored at $3^{\circ} \mathrm{C}$ for 6 mo was similar in flavor to MPC 45 stored at $25^{\circ} \mathrm{C}$ for $3 \mathrm{mo}$ and MPC 45 stored at $40^{\circ} \mathrm{C}$ for 1 mo, whereas MPC 45 stored at $25^{\circ} \mathrm{C}$ stored for 6 mo had a similar flavor profile to MPC 45 stored at $40^{\circ} \mathrm{C}$ for 3 mo. It is important to note that MPC 45 stored at $3^{\circ} \mathrm{C}$ for 12 mo never developed the off-flavor profile of MPC 45 stored at $25^{\circ} \mathrm{C}$ by 6 mo and MPC 45 stored at $40^{\circ} \mathrm{C}$ by 3 mo. Milk protein concentrate (45\%) stored at $40^{\circ} \mathrm{C}$ for 6 mo was more highly associated with tortilla, cardboard, animal, and burnt sugar flavors than MPC 45 stored at $25^{\circ} \mathrm{C}$ for $12 \mathrm{mo}$.

The effect of storage temperature on the flavor of MPC 80 was more pronounced than its effect on MPC 45. The MPC 80 stored at $3^{\circ} \mathrm{C}$ for 12 mo was similar in flavor to MPC 80 stored at $25^{\circ} \mathrm{C}$ for $6 \mathrm{mo}$ and MPC 80 stored at $40^{\circ} \mathrm{C}$ for 1 mo, whereas MPC 80 stored at $25^{\circ} \mathrm{C}$ for $12 \mathrm{mo}$ had a similar flavor profile to MPC 45 stored at $40^{\circ} \mathrm{C}$ for $3 \mathrm{mo}$. The effect of temperature is especially important to consider during transport over- 
Biplot (axes F1 and F2: 73 \%)

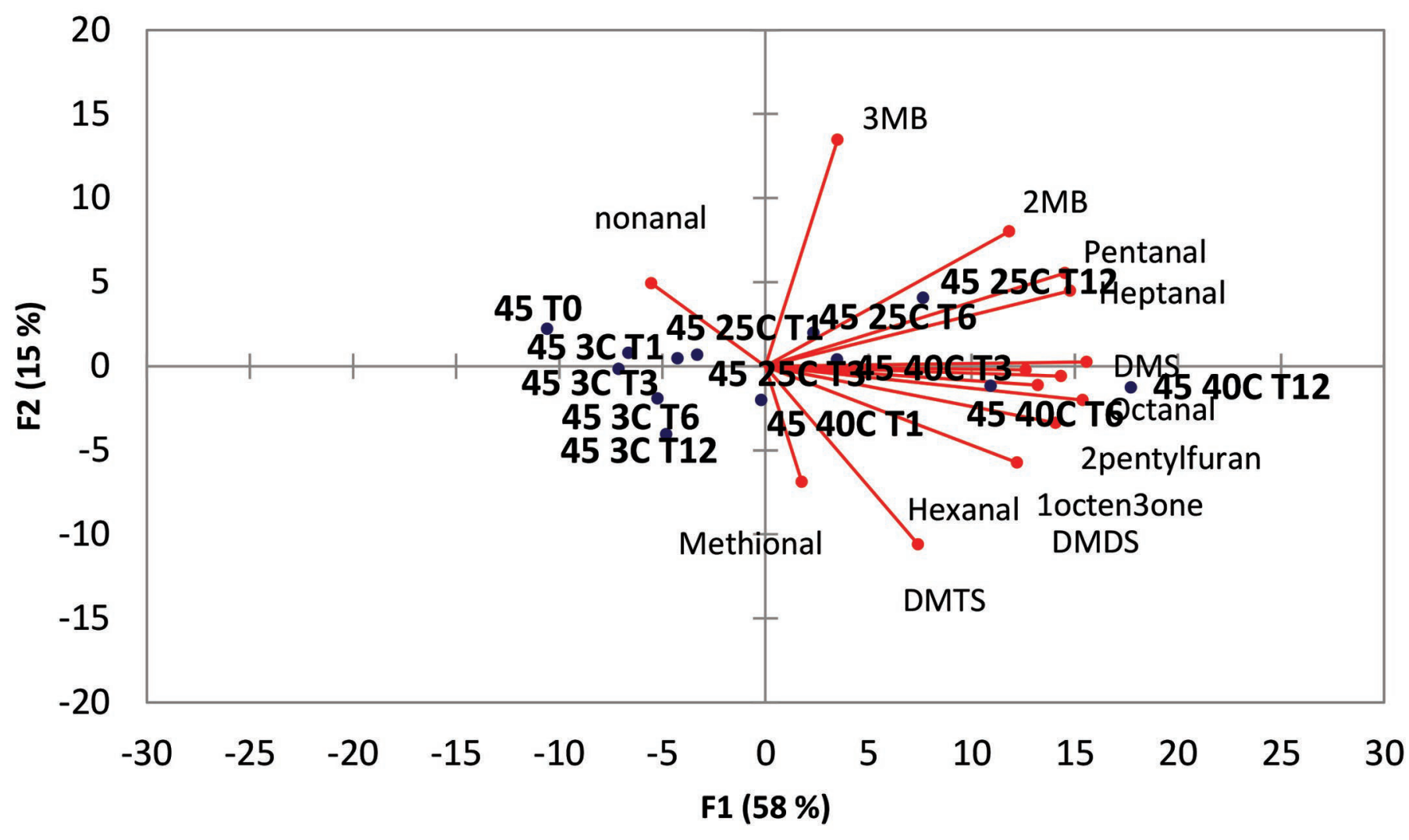

Figure 6. Principal component biplot of relative abundance of selected volatile compounds of milk protein concentrate (MPC) 45 stored at 3 , 25 , and $40^{\circ} \mathrm{C}$ for $0,1,3,6$, and $12 \mathrm{mo} . \mathrm{T} 0=$ no storage, $\mathrm{T} 1=1$ mo storage, $\mathrm{T} 3=3$ mo storage, $\mathrm{T} 6=6$ mo storage, $\mathrm{T} 12=12 \mathrm{mo}$ storage. $3 \mathrm{C}=$ stored at $3{ }^{\circ} \mathrm{C}, 25 \mathrm{C}=$ stored at $25^{\circ} \mathrm{C}, 40 \mathrm{C}=$ stored at $40^{\circ} \mathrm{C}$. DMDS $=$ dimethyl disulfide; DMTS $=$ dimethyl trisulfide; $2 \mathrm{MB}=2$ methylbutanal; $3 \mathrm{MB}=3$ methylbutanal. Color version available online.

seas, when powder may be stored at high temperatures for extended periods. The flavor related shelf life of MPC 45 stored at high temperatures $\left(40^{\circ} \mathrm{C}\right)$ is half that of those stored at lower temperatures $\left(25^{\circ} \mathrm{C}\right)$, whereas the shelf life of MPC 80 stored at high temperatures $\left(40^{\circ} \mathrm{C}\right)$ is a quarter of those stored at $25^{\circ} \mathrm{C}$. Refrigerated storage $\left(3^{\circ} \mathrm{C}\right)$ of MPC greatly decreases the development of off flavors but does not completely suspend it.

Previous studies on MPC have focused on functionality, primarily solubility, rather than instrumental volatile and sensory properties. Whereas a few studies have been published on sensory properties of milk proteins, to the authors' knowledge, the effect of storage on milk protein flavor has not been previously addressed. Compounds associated with lipid oxidation and Maillard browning are the primary source of flavor in milk proteins and storage increased the concentration of many of these compounds. Although lipid content in the powders is relatively low, lipid oxidation products have a low odor threshold and are well established as contributors to off flavor in dried dairy ingredients (Carunchia Whetstine et al., 2005; Drake et al., 2009). Aldehydes (hexanal, heptanal, octanal, nonanal, methional, 2 and 3-methylbutanal, E,E 2,4 decadienal) and other lipid oxidation products such as 2-pentylfuran and 1-octen-3-one and sulfur-containing volatile compounds increased over time and as storage temperature increased in both MPC 45 and MPC 80. Drake et al. (2006) compared flavor stability of SMP stored at 21 or $35^{\circ} \mathrm{C}$, and Wright et al. (2009) studied the flavor stability of whey protein concentrates $(80 \%)$ and isolates across 18 mo storage at $21^{\circ} \mathrm{C}$. Wright et al. (2009) documented that lipid oxidation increased during storage of whey proteins, confirming the involvement of lipid oxidation in dried dairy protein ingredients during storage. Drake et al. (2006) also documented lipid oxidation compounds in SMP stored at $21^{\circ} \mathrm{C}$ as well as increased Maillard reaction production in SMP stored at $35^{\circ} \mathrm{C}$. They documented that different flavors as well as different volatile compounds formed in milk powders 
stored at different temperatures. These results collectively are consistent with the results from the current study with MPC.

\section{CONCLUSIONS}

Flavor is of utmost importance to the success of milk protein products. The flavor of different milk protein products varied depending on the composition, manufacturing process, and storage. Milk proteins were characterized by sweet aromatic, sour aromatic, cardboard, potato/brothy, brothy/animal, tortilla/corn chip, soapy, and fatty flavors. Tortilla flavor is a defining flavor of milk protein ingredients and many of the odor active volatile compounds found in corn tortillas, corn chips, and taco shells were also odor active compounds in milk proteins. Key flavor active compounds in milk proteins were 1-hexen-3-one, 2-acetyl-1-pyrroline, benzothiazole, 1-octadecanol, homofuraneol, 1-octen-3-one, 1-hexen-3-one, sotolon, homofuraneol, 4-hexen-3-one, 1-cyclohexen-3-one, dimethyl disulfide, dimethyl trisulfide, guaiacol, methional, hexanal, heptanal, octanal, nonanal, decanal, 2-aminoacetophenone, acetic, propionic, butanoic, pentanoic, and hexanoic acids, gamma-decalactone, and delta-dodecalactone. The MPC 45 and 80 stored at lower temperatures for less time were associated with sweet aromatic and cooked milky flavors and a sweet taste. As the powders aged, aroma intensity, cardboard, animal, burnt sugar, and tortilla/corn chip flavors increased. Storage temperature played a large role in the shelf life of MPC. At high temperature $\left(40^{\circ} \mathrm{C}\right), 1$ mo storage was sufficient to reduce solubility of MPC 45 and 80 below the solubility of 3 and $25^{\circ} \mathrm{C}$ stored MPC 45 and 80 . Storage at high temperatures $\left(40^{\circ} \mathrm{C}\right)$ also reduced the flavor related shelf life of MPC 45 by $50 \%$, and by $75 \%$ in MPC 80 compared with MPC 45 and 80 stored at $25^{\circ} \mathrm{C}$. Based on sensory and functional changes, shelf life of MPC at temperatures greater than $40^{\circ} \mathrm{C}$ is less than $3 \mathrm{mo}$, up to $6 \mathrm{mo}$ at $25^{\circ} \mathrm{C}$, and more than $12 \mathrm{mo}$ at $3^{\circ} \mathrm{C}$. Special care must be taken during shipping and storage or the effective shelf-life of MPC is drastically reduced.

\section{Biplot (axes F1 and F2: 87 \%)}

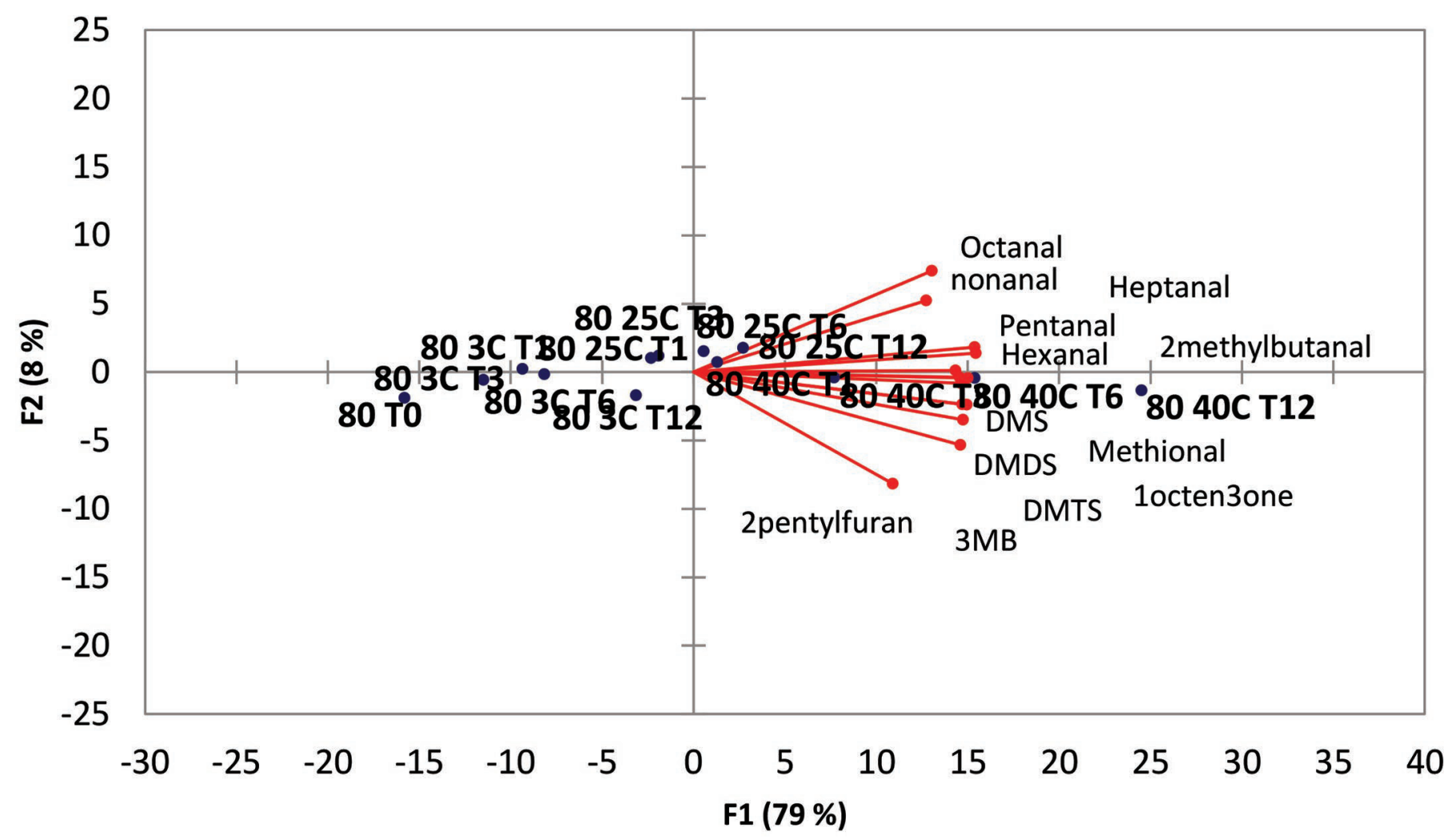

Figure 7. Principal component biplot of relative abundance of selected volatile compounds of milk protein concentrate (MPC) 80 stored at 3,25 , and $40^{\circ} \mathrm{C}$ for $0,1,3,6$, and 12 mo. $\mathrm{T} 0=$ no storage, $\mathrm{T} 1=1$ mo storage, $\mathrm{T} 3=3$ mo storage, $\mathrm{T} 6=6$ mo storage, $\mathrm{T} 12=12$ mo storage. $3 \mathrm{C}=$ stored at $3{ }^{\circ} \mathrm{C}, 25 \mathrm{C}=$ stored at $25^{\circ} \mathrm{C}, 40 \mathrm{C}=$ stored at $40^{\circ} \mathrm{C}$. DMS $=$ dimethyl sulfide; DMDS $=$ dimethyl disulfide; DMTS $=$ dimethyl trisulfide; $2 \mathrm{MB}=2$ methylbutanal; $3 \mathrm{MB}=3$ methylbutanal. Color version available online. 


\section{ACKNOWLEDGMENTS}

Funding was provided in part by the National Dairy Council (Rosemont, IL). The use of tradenames does not imply endorsement of products nor lack of endorsement of those not mentioned.

\section{REFERENCES}

ADPI. 2015. Milk Protein Isolate. Accessed May 16th, 2015. https:// www.adpi.org/DairyProducts/DryMilks/MilkProteinIsolate/ tabid/358/Default.aspx.

Anema, S. G., D. N. Pinder, R. J. Hunter, and Y. Hemar. 2006. Effects of storage temperature on the solubility of milk protein concentrate (MPC85). Food Hydrocoll. 20:386-393.

Arnold, R. G., L. M. Libbey, and E. A. Day. 1966. Identification of components in the stale flavor fraction of sterilized concentrated milk. J. Food Sci. 31:566-573.

Azzara, C. D., and L. B. Campbell. 1992. Off-flavors of dairy products. Pages 329-374 in Off-Flavors in Food and Beverages. G. Charalambous, ed. Elsevier Science Publishers, Amsterdam, the Netherlands.

Badings, H. T. 1991. Milk. Pages 91-106 in Volatile Compounds in Foods and Beverages. H. Maarse, ed. Marcel Dekker, New York, NY.

Baldwin, A. J., and G. N. T. Truong. 2007. Development of insolubility in dehydration of dairy milk powders. Food Biol. Process. 85:202-208.

Baptista, J. A. B., and R. C. C. Carvalho. 2004. Indirect determination of Amadori compounds in milk-based products by HPLC/ ELSD/UV as an index of protein deterioration. Food Res. Int. 37:739-747.

Biemel, K. M., H. P. Buhler, O. Reihl, and M. O. Lederer. 2001. Identification and quantitative evaluation of the lysine-arginine crosslinks GODIC, MODIC, DODIC, and glucosepan in foods. Nahrung/Food 45:210-214.

Buttery, R. G., and L. C. Ling. 1995. Volatile flavor components of corn tortillas and related products. J. Agric. Food Chem. 43:18781882 .

Buttery, R. G., and L. C. Ling. 1998. Additional studies on flavor components of corn tortilla chips. J. Agric. Food Chem. 46:2764-2769.

Buttery, R. G., D. J. Stern, and L. C. Ling. 1994. Studies on flavor volatile of some sweet corn products. J. Agric. Food Chem. 42:791-795.

Campbell, R. E., M. C. Adams, M. A. Drake, and D. M. Barbano. 2013. Effect of bleaching permeate from microfiltered skim milk on $80 \%$ serum protein concentrate. J. Dairy Sci. 96:1387-1400.

Campbell, R. E., R. E. Miracle, and M. A. Drake. 2011. The effect of starter culture and annatto on the flavor and functionality of whey protein concentrate. J. Dairy Sci. 94:1185-1193.

Carunchia Whetstine, M. E., A. E. Croissant, and M. A. Drake. 2005. Characterization of dried whey protein concentrate and isolate flavor. J. Dairy Sci. 88:3826-3839.

Carunchia Whetstine, M. E., Y. Karagul-Yuceer, Y. K. Avsar, and M. A. Drake. 2003. Identification and quantification of character aroma components in fresh chevre-style goat cheese. J. Food Sci. 68:2441-2447.

Croissant, A. E., E. J. Kang, R. E. Campbell, E. Bastian, and M. A. Drake. 2009. The effect of bleaching agent on the flavor of liquid whey and whey protein concentrate. J. Dairy Sci. 92:5917-5927.

Dalsgaard, T. K., D. Otzen, J. H. Nielson, and L. B. Larson. 2007. Changes in structures of milk proteins upon photo-oxidation. J. Agric. Food Chem. 55:10968-10976.

Drake, M. A., R. E. Miracle, A. D. Caudle, and K. R. Cadwallader 2006. Relating sensory and instrumental analyses. Pages 23-55 in Sensory-Directed Flavor Analysis. R. Marsili, ed. CRC Press, Taylor and Francis Publishing, Boca Raton, FL.

Drake, M. A., Y. Karagul-Yuceer, K. R. Caldwallader, G. V. Civille, and P. S. Tong. 2003. Determination of the sensory attributes of dried milk powders and dairy ingredients. J. Sens. Stud. 18:199 216.

Drake, M. A., R. E. Miracle, and J. M. Wright. 2009. Sensory properties of dairy proteins. Pages 429-448 in Milk Proteins: From Expression to Food. A. Thompson, M. Boland, and H. Singh, ed. Elsevier, New York, NY

Erbersdobler, H. F. 1986. Amino carbonyl reactions in food and biological systems. Pages 481-491 in Proceedings of the 3rd International Symposium on the Maillard Reaction. M. Fujimaki, M. Namiki, and H. Kato, ed. Elsevier, Amsterdam, the Netherlands.

Evans, J., J. Zulewska, M. Newbold, M. A. Drake, and D. M. Barbano. 2009. Comparison of composition, sensory and volatile components of thirty-four percent whey protein and serum protein concentrates. J. Dairy Sci. 92:4773-4791.

Fox, A. J., T. J. Smith, P. D. Gerard, and M. A. Drake. 2013. The influence of bleaching agent and temperature on bleaching efficacy and volatile components of fluid whey and whey retentate. J. Food Sci. 78:C1535-C1542.

Grosch, W., and P. Schieberle. 1997. Flavor of cereal products-A review. J. Cereal Chem. 74:91-97.

Hammond, E. G., and F. D. Hill. 1964. The oxidized-metallic and grassy flavor components of autoxidized milk fat. J. Am. Oil Chem. Soc. 3:180-184

Havea, P. 2006. Protein interactions in milk protein concentrate powders. Int. Dairy J. 16:415-422.

Jervis, S., R. E. Campbell, K. L. Wojciechowski, M. A. Drake, and D. M. Barbano. 2012. Impact of bleaching whey on sensory and functional properties of $80 \%$ whey protein concentrate. J. Dairy Sci. 95:2848-2862.

Karagul-Yuceer, M. A. D., and K. R. Cadwallader. 2001. Aroma-active components of nonfat dry milk. J. Agric. Food Chem. 49:2948 2953.

Karagul-Yuceer, Y., K. R. Cadwallader, and M. A. Drake. 2002. Volatile flavor components of stored nonfat dry milk. J. Agric. Food Chem. 50:305-312.

Karagul-Yuceer, Y., K. N. Vlahovich, M. A. Drake, and K. R. Cadwallader. 2003. Characteristic aroma compounds of rennet casein. J. Agric. Food Chem. 51:6797-6801

Karahadian, C., and K. A. Johnson. 1993. Analysis of headspace volatiles and sensory characteristics of fresh corn tortillas made from fresh masa dough and spray-dried masa flour. J. Agric. Food Chem. 41:791-799.

Karatapanis, A. E., A. V. Badeka, K. A. Riganakos, I. N. Savvaidis, and M. G. Kontominas. 2006. Changes in flavor volatiles of whole pasteurized milk as affected by packaging material and storage time. Int. Dairy J. 16:750-761.

Kobayashi, N., Y. Mizota, K. Kumazawa, and O. Nishimura. 2008. Character impact odorants of high-heat skim milk powder isolated by simultaneous distillation-extraction. J. Agric. Food Chem. 56:2469-2476.

Le, T. T., B. Bhandari, and H. C. Deeth. 2011a. Chemical and physical changes in milk protein concentrate (MPC80) powder during storage. J. Agric. Food Chem. 59:5465-5473.

Le, T. T. B. Bhandari, J. W. Holland, and H. C. Deeth. 2011b. Maillard reaction and protein cross-linkingin relation to the solubility of milk powders. J. Agric. Food Chem. 59:12473-12479.

Le, T. T., J. W. Holland, B. Bhandari, P. F. Alewood, and H. C. Deeth. 2013. Direct evidence for the role of Maillard reaction products in protein cross-linking in milk powder during storage. Int. Dairy J. 31:83-91.

Lederer, M. O., and R. G. Klaiber. 1999. Cross-linking of proteins by Maillard processes: Characterization and detection of lysine-arginine cross-links derived from glyoxal and methylglyoxal. Bioorg. Med. Chem. 7:2499-2507.

Leksrisompong, P. P., R. E. Miracle, and M. A. Drake. 2010. Characterization of flavor of whey protein hydrolysates. J. Agric. Food Chem. 58:6318-6327.

Liaw, I., H. Eshpari, P. S. Tong, and M. A. Drake. 2010. The impact of antioxidant addition on flavor of Cheddar and Mozzarella whey and Cheddar whey protein concentrate. J. Food Sci. 75:C559C569. 
Liaw, I. W., R. E. Miracle, S. M. Jervis, M. A. D. Listiyani, and M. A. Drake. 2011. Comparison of the flavor chemistry and flavor stability of mozzarella and Cheddar wheys. J. Food Sci. 76:C1188C1194.

Lloyd, M. A., S. J. Hess, and M. A. Drake. 2009. Effect of nitrogen flushing and storage temperature on flavor and shelf-life of whole milk powder. J. Dairy Sci. 92:2409-2422.

Mahajan, S. S., L. Goddik, and M. C. Qian. 2004. Aroma compounds in sweet whey powder. J. Dairy Sci. 87:4057-4063.

Manley, C. H., and I. S. Fagerson. 1970. Major volatile components of the basic fraction of hydrolyzed soy protein. J. Agric. Food Chem. 18:340-342.

Mao, X. Y., P. S. Tong, S. Guaico, and S. Vink. 2012. Effect of NaC addition during diafiltration on the solubility, hydrophobicity and disulfide bonds of $80 \%$ milk protein concentrate powder. J. Dairy Sci. 95:3481-3488.

Meilgaard, M. C., G. V. Civille, and B. T. Carr. 2009. The Spectrum ${ }^{\mathrm{TM}}$ descriptive analysis method. Pages 189-253 in Sensory Evaluation Techniques. CRC Press, Boca Raton, FL.

Parks, O. W., D. P. Schwartz, and M. Keeney. 1964. Identification of 2-aminoacetophenone as a flavor compound in stale dry milk. Nature 202:185-187.

Parks, O. W., D. P. Schwartz, K. Nelson, and C. Allen. 1967. Evidence for kynurenine in milk. J. Dairy Sci. 50:10-11.

Preininger, M., and W. Grosch. 1994. Evaluation of key odorants of the neutral volatiles of Emmentaler cheese by the calculation of odour activity values. Food Sci. Technol. (Campinas.) 27:237-244.

Ramshaw, E. H., and E. A. Dunstone. 1969. Volatile compounds associated with the off-flavor in stored casein. J. Dairy Res. 36:215223.

Schieberle, P. 1995. Quantitation of important roast-smelling odorants in popcorn by stable isotope dilution assays and model studies on flavor formation during popping. J. Agric. Food Chem. 43:24422448.

Schuck, M., S. Piot, S. Mejean, J. Fauquant, G. Brule, and J. L. Maubois. 1994. Dehydration of an ultra-clean milk and micellar casein enriched milks. Lait 74:47-63.

Shiratsuchi, H., M. Shimoda, K. Imayoshi, K. Noda, and Y. Osajima. 1994a. Volatile flavor compounds in spray-dried skim milk powder. J. Agric. Food Chem. 42:984-988.

Shiratsuchi, H., M. Shimoda, K. Imayoshi, K. Noda, and Y. Osajima. 1994b. Off-flavor compounds in spray-dried skim milk powder. J. Agric. Food Chem. 42:1323-1327.
Sikand, V., P. S. Tong, and J. Walker. 2013. Effect of adding salt during the diafiltration step of milk protein concentrate powder manufacture on mineral and soluble protein composition. Dairy Sci. Technol. 93:401-413.

Smith, T. J., R. E. Campbell, and M. A. Drake. 2016. Sensory properties of milk protein ingredients. pp. 197-223 in Advanced Dairy Chemistry, Volume 1B: Proteins: Applied Aspects. P. L. H. McSweeney and P. F. Fox, ed. Springer. New York, NY.

Spacek, M. 1954. Simultaneous determination of kynurenine and pphenetidine in human urine. Can. J. Biochem. Physiol. 32:604-609.

Spies, J. R. 1967. Determination of tryptophan in proteins. Anal Chem. 39:1412-1416.

Tabone, J., N. Mamounas, and D. Robert. 1951. Kynurenine as a precursor of 2-aminoacetophenone during the decomposition of tryptophan by dilute alkalies. Bull. Soc. Chim. Biol. (Paris) $33: 1560-1563$

Thomas, M. E. C., J. Scher, S. Desobry-Banon, and S. Desobry. 2004. Milk powders ageing: effect on physical and functional properties. Crit. Rev. Food Sci. Nutr. 44:297-322.

Tressl, R., B. Helak, N. Martin, and E. Kersten. 1989. Formation of amino acid specific Maillard products and their contribution to thermally generated aromas, in, Thermal Generation of Aromas. T. H. Parliament, R. J. McGorrin, and C. Ho, ed. ACS Symposium Series 409. American Chemical Society, Washington, DC.

USDEC. 2012. Opportunities for milk protein concentrate Accessed Oct. 17, 2013. www.usdec.org/files/exportprofile/ exportprofileaug2012.pdf.

Vandendool, H., and P. Kratz. 1963. A generalization of the retention index system including linear programmed gas liquid partition chromatography. J. Chromatogr. 11:463-471.

Whitson, M. E., R. E. Miracle, E. Bastian, and M. A. Drake. 2011 Effect of liquid retentate storage on flavor of spray dried whey protein concentrates and isolate. J. Dairy Sci. 94:3747-3760.

Whitson, M. E., R. E. Miracle, and M. A. Drake. 2010. Sensory characterization of chemical components responsible for cardboard flavor in whey protein. J. Sens. Stud. 25:616-636

Wright, B. J., S. E. Zevchak, J. M. Wright, and M. A. Drake. 2009. The impact of agglomeration and storage on flavor and flavor stability of whey protein concentrate 80 and whey protein isolate. J. Food Sci. 74:S17-S29.

Wright, J. M., M. E. Carunchia Whetstine, R. E. Miracle, and M. A Drake. 2006. Characterization of cabbage off-flavor in whey protein isolate. J. Food Sci. 71:C86-C90. 